\title{
Ginsenoside Rg1 Reduced Microglial Activation and Mitochondrial Dysfunction to Alleviate Depression-Like Behaviour Via the GAS5/ EZH2/SOCS3/NRF2 Axis
}

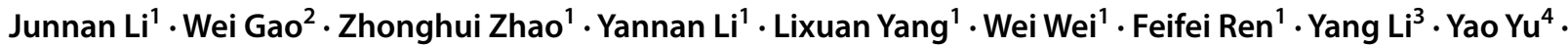 \\ Wenzhe Duan ${ }^{1} \cdot$ Jingchun $\mathrm{Li}^{1} \cdot$ Baoan Dai ${ }^{1} \cdot$ Rongjuan Guo $^{5}$
}

Received: 14 October 2021 / Accepted: 7 January 2022 / Published online: 1 March 2022

(c) The Author(s) 2022

\begin{abstract}
Ginsenoside $\mathrm{Rg} 1$ is the principal active ingredient in ginseng. The antidepressant effects of $\mathrm{Rg} 1$ have been validated; however, the specific underlying mechanism of this effect needs further research. Rats were subjected to the chronic restraint stress (CRS) depression model. Rg1, or a positive control drug, was administered to the rats. Depression-like behaviours were evaluated through behavioural experiments. Cytokine, mRNA, protein, ATP, and mitochondria DNA levels were detected using the indicated methods. Lentivirus-packaged plasmids were injected into the rat brain for GAS5 overexpression or knockdown. In vitro mitochondrial dysfunction was evaluated by detecting mitochondrial reactive oxygen species and mitochondrial membrane potential. Direct interaction between GAS5 and EZH2 was validated by RNA immunoprecipitation and RNA pulldown assay. The enrichment of EZH2 and H3K27me3 was evaluated through chromatin immunoprecipitation quantitative real-time PCR. Rg1 treatment alleviated depression-like behaviours, microglial activation, and mitochondrial dysfunction in CRS rats. Similarly, GAS5 knockdown revealed a similar protective effect of Rg1 treatment. GAS5 overexpression in the rat brain compromised the protective effect of $\mathrm{Rg} 1$ treatment. Moreover, $\mathrm{Rg} 1$ treatment or GAS5 knockdown attenuated microglial activation and mitochondrial dysfunction in vitro. Mechanically, GAS5 was suppressed SOCS3 and NRF2 expression by facilitating EZH2-mediated transcriptional repression. Rg1 attenuated microglial activation and improved mitochondrial dysfunction in depression by downregulating GAS5 expression. Mechanically, GAS5 might regulate microglial activation and mitochondrial dysfunction via the epigenetic suppression of NRF2 and SOCS3.
\end{abstract}

Keywords Ginsenoside Rg1 · GAS5 · EZH2 · Depression · SOCS3 · NRF2

Rongjuan Guo

dfguorongjuan@163.com

1 Second Clinical Medical College, Beijing University of Chinese Medicine, Beijing 100029,

People's Republic of China

2 Tsinghua University, Yuquan Hospital, Beijing 100040, People's Republic of China

3 Beijing Hospital of Traditional Chinese Medicine, Capital Medical University, Beijing 100010, People's Republic of China

4 Beijing Changping Hospital of Integrated Chinese and Western Medicine, Beijing 102208, People's Republic of China

5 Department of Neurology, Dongfang Hospital Beijing University of Chinese Medicine, No. 6 Fangxingyuan 1st Block, Fengtai District, Beijing 100078, People's Republic of China

\section{Abbreviations}

CRS Chronic restraint stress

SSRIs Selective 5-HT reuptake inhibitors

CNS

CMS

ROS

Central nervous system

Chronic mild stress

GAS5

Reactive oxygen species

EZH2

Growth arresting-specific 5

$\mathrm{PRC} 2$

Enhancer of zeste homolog 2

H3K27me3 Methylation of histone 3 on lysine 27

SOCS3 Suppressor of cytokine signaling 3

Nrf2 


\section{Introduction}

Depression is a type of emotional disorder that brings a tremendous burden to society and individuals. Depression is characterized by high morbidity, mortality, and disability rates [1]. Typical symptoms of depression include depressed mood, retardation of thought, loss of volitional activity, and cognitive impairment [2]. So far, the cause of depression remains elusive. Several biological, psychological, and social environmental factors are associated with the pathogenesis of depression. Biological factors mainly involve heredity, neurobiochemical, neuroendocrine, and nerve regeneration [3]. Currently, most antidepressants in clinical use are synthetic compounds, including selective 5-HT reuptake inhibitors (SSRIs) [4]. Although the current treatments improve depression, they fail to resolve the symptoms entirely in more than half of the cases. Thus, it is urgent to identify novel potential candidates for the treatment of depression.

Microglia, a type of glial cell, are macrophages in the brain and spinal cord. As the primary immune line of defense in the central nervous system (CNS), microglia clears the damaged nerves and infectious substances in the CNS [5]. However, overactivated microglia can cause neurotoxicity by releasing pro-inflammatory cytokines [6]. Several studies have reported that the overactivation of microglia plays a crucial role in the pathogenesis of neurodegenerative diseases, including Parkinson's and Alzheimer's diseases [7, 8]. Notably, microglia are involved in the pathogenesis of depression [9]. Overactivation of microglia and upregulation of inflammatory cytokines have been observed in depression models [10]. Moreover, growing evidence has indicated that inhibiting the overactivation of microglia can ameliorate depressionlike behaviour. Bassett et al. reported that minocycline attenuated depression-like behaviour in chronic mild stress (CMS)-induced mice via suppressing the overactivation of microglia [11]. Melatonin can also attenuate depressionlike behaviours by inhibiting microglial activation [12]. Hence, targeting microglial activation may be an effective manner to treat depression.

Mitochondria are the principal organelles of cellular energy metabolism and reactive oxygen species (ROS) generation [13]. Recently, an increasing number of studies have reported that mitochondrial function and structure are abnormal in patients with depression, and changes in the mitochondrial ultrastructure can lead to energy metabolism disorders. Therefore, mitochondrial dysfunction is proposed to be involved in the pathogenesis of depression [14]. Depression is usually associated with an increase in intracellular ROS levels and a decrease in antioxidant capacity. As the primary production site of cellular ROS, mitochondria are sensitive to oxidative stress injury. Mitochondrial dysfunction can further aggravate oxidative damage [15]. A study by Wang et al. suggested that injecting isolated mitochondria abrogated microglial activation, suppressed ROS generation, and ameliorated depression-like behaviours [16]. Hence, discovering the significance of mitochondrial dysfunction in the pathogenesis of depression might help generate novel therapeutic targets for depression treatment.

Ginsenoside Rg1 is a steroid compound, also known as triterpenoid saponin, primarily found in Panax ginseng and is regarded as the active ingredient in ginseng. Numerous studies have demonstrated the protective role of $\mathrm{Rg} 1$ in neurological diseases, including cerebral ischemia, Alzheimer's disease, and Parkinson's disease [17-19]. Additionally, growing evidence has revealed the protective role of $\mathrm{Rg} 1$ in depression. Rg1 reportedly abrogates oxidative stress and inflammation in the neurons to alleviate stress-induced depression-like behaviours [20]. Another study reported that Rg1 activates the BDNF signaling pathway and promotes neurogenesis [21].

Moreover, a study by $\mathrm{Yu}$ et al. revealed that $\mathrm{Rg} 1$ might exert its protective role in depression via the regulation of neuronal structural plasticity [22]. Although the functional role of $\operatorname{Rg} 1$ has been demonstrated in depression, it remains unclear whether Rg1 could exhibit its protective effect by improving mitochondrial function. In addition, the underlying molecular mechanism of $\mathrm{Rg} 1$ in depression also remains largely unknown.

Long non-coding RNAs (lncRNAs) are RNAs with more than 200 bases in length that cannot encode proteins [23]. Recently, lncRNAs have been demonstrated to play a crucial role in the pathogenesis of depression. A study on the analysis of lncRNA expression in peripheral blood leukocytes of patients with depression revealed that lncRNA RMRP in the patients was significantly reduced, which might be used as a depression marker [24]. A functional study revealed that lncRNA uc.80 switches microglia polarization to M2 subtype and ameliorate depression-like behaviours in rats [25]. Additionally, lncRNA MIR155HG inhibits depression-like behaviours by upregulating BDNF expression [26]. lncRNA growth arresting-specific 5 (GAS5) has been proven to be involved in various physiological and pathological processes. Specifically, inhibiting lncRNA GAS5 can improve depression-like behaviours by reducing neuronal damage in the hippocampus [27]. However, the underlying mechanism must be further investigated.

In the present study, we aimed to verify the protective role of Rg1 in depression. Rg1 attenuated microglial activation and improved mitochondrial dysfunction by downregulating the expression of GAS5. The protective role of GAS5 inhibition was also proven in the depression model. Mechanically, GAS5 regulated microglial activation and mitochondrial dysfunction via the epigenetic suppression of NRF2 and SOCS3. Our result might provide novel insight for understanding the role of $\mathrm{Rg} 1$ in the treatment of depression. 


\section{Materials and Methods}

\section{Animals}

Male SD rats (weight: $200-250 \mathrm{~g}$ ) were purchased from Charles River Laboratories (Beijing, China) and maintained at $22^{\circ} \mathrm{C} \pm 1{ }^{\circ} \mathrm{C}$ at a diurnal cycle of $12-\mathrm{h}$ and were provided with free access to water and food. All animal experiments were conducted in strict adherence to the China-Japan Friendship Hospital guidelines on the care and use of laboratory animals. The study was approved by the Ethics Committee of China-Japan Friendship Hospital (Approval number: zryhyy 21-20-09-9).

\section{Plasmid Construction and Transfection}

Sh-GAS5 and the negative-control shRNA (sh-NC) were synthesized by GenePharma (Shanghai, China), and cloned into a pLKO.1 vector. The pLKO.1-shGAS5 plasmids were then transfected into HEK293T cells with a psPAX2 packaging plasmid using the Lipofectamine 3000 to produce shRNA-containing lentivirus (lenti-shRNA). The lentishRNA was then used to infect cells or microinjected into an animal ventricle to construct GAS5 silent rats.

A GAS5-overexpressing plasmid was generated using a full-length GAS5 that was cloned and inserted into multiple cloning sites of the pLenti6.3/V5-DEST expression plasmid and then transfected into $293 \mathrm{~T}$ cells using the Lipofectamine 3000 , together with $\mathrm{pLP} 1$ and $\mathrm{pLP} 2$ packaging plasmids and pLP/VSVG envelope plasmid, to produce GAS5-expressing lentivirus. The resultant lentivirus was used to generate GAS5-overexpressing (lenti-GAS5) rats or cells.

\section{Chronic Restraint Stress Model}

Before chronic restraint stress (CRS) modeling, 1 week (days 1-3: adaptation to the environment; days 4-5: 2 bottles of pure water; days 6-7: a bottle of $1 \%$ sucrose water and a bottle of fresh water) was allowed for adaptation purpose. The rats in CRS group were then restricted in an adjustable cylindrical plastic bound pipe (made by $50 \mathrm{ml}$ centrifuge tubes with pre-blistered air holes dispersed throughout the tube and a small hole in the center of the cap through which the mouse's tail could be exposed to the air) fitted to allow them to breathe for $4 \mathrm{~h}$ every day (from 09:00 to 13:00) with no food and water for 28 consecutive days. A group of rats (control) was not subjected to any treatment other than restraining food and water supply during 09:00-13:00 to match the test rats.

The animal treatment $(n \geq 5)$ of the experiments is depicted in Figs. 1 and 2: for CRS + Rg1/escitalopram group, $\operatorname{Rg} 1$ (20 mg/kg/day) or escitalopram ( $1 \mathrm{mg} / \mathrm{kg} /$ day $)$ was administered to the rats via the intragastric route $1 \mathrm{~h}$ after CRS. Meanwhile, an equal amount of fresh water was provided to the control and CRS group of rats. As depicted in Figs. 3 and 4, for lenti-shRNA injected group, rats were anesthetized with 5\% chloral hydrate by intraperitoneal (i.p.) injection and placed on a stereotaxic apparatus. Small holes were drilled into the skull, and lenti-shRNA ( $5 \mu \mathrm{L}$ of $10^{8}$ viral genome $/ \mu \mathrm{L}$; HANBIO, Shanghai, China) was microinjected into the lateral ventricle at the following coordinates: $0.3 \mathrm{~mm}$ behind the bregma and $1.0 \mathrm{~mm}$ lateral from the sagittal midline, at a depth of $2.2 \mathrm{~mm}$ from the skull surface on the last day of adaptation by using a Nanoject II (Drummond) system at a rate of $0.1 \mu \mathrm{l}$ per min. The injection cannula was slowly withdrawn 5 min after the virus infusion. The scalp was then sealed, and injected mice were monitored as they recovered from anesthesia. Five microliter of normal saline was microinjected into the rats in the control and CRS groups, as mentioned above. As shown in Fig. 5, for the CRS + Rg1 + lenti-RNA group, the microinjection and $\operatorname{Rg} 1$ treatment methods were kept the same as mentioned above. Rats were recovered for $48 \mathrm{~h}$ before CRS modeling. $\mathrm{Rg} 1$ (HY-N0045) and escitalopram (HY-14258) were purchased from MedChemExpress.

\section{Cell Culture}

The PC-12 cell line was purchased from the National Collection of Authenticated Cell Cultures (China, Shanghai) and the HAPI cell line from BeNa Culture Collection (Beijing, China). PC-12 cells were cultured in RPMI 1640 medium (Gibco, USA) and the HAPI cells in the DMEM/F-12 medium (Gibco). All culture systems were supplemented with $10 \%$ fetal bovine serum (FBS) and $1 \%$ penicillin-streptomycin (Gibco) under a humidified atmosphere of $5 \% \mathrm{CO}_{2}$ at $37^{\circ} \mathrm{C}$.

The cell treatment of the experiments is depicted in Figs. 6 and 7 and was as follows: for the LPS/corticosterone $+\operatorname{Rg} 1$ group, the cells were pre-treated with LPS $(1 \mu \mathrm{g} /$ $\mathrm{mL}$, to establish an inflammatory stress model) or corticosterone ( $400 \mu \mathrm{M}$, to establish an oxidative stress model) for $2 \mathrm{~h}$, followed by co-incubation with $\operatorname{Rg} 1(5 \mu \mathrm{M}, 10 \mu \mathrm{M}$, $20 \mu \mathrm{M}$ ) for $24 \mathrm{~h}$. For the LPS/corticosterone + shRNA group, the cells were transfected with lentivirus for $48 \mathrm{~h}$ and then washed with cold PBS buffer, followed by incubation with LPS or corticosterone for $24 \mathrm{~h}$. Corticosterone (HY-B1618) was purchased from MedChemExpress and LPS (L5293) from Sigma-Aldrich.

\section{Sucrose Preference Test}

The sucrose preference test (SPT) was performed for $24 \mathrm{~h}$ on the last day after CRS modeling. Two bottles of water were offered to the rats, one containing fresh water and the other 
containing $1 \%$ sucrose, and the positions of the two bottles were switched once to avoid the (left/right) position preference. The sucrose preference index was calculated as sucrose consumption/total liquid consumption $\times 100 \%$.

\section{Open Field Test}

The open field test (OFT) was performed after SPT. Briefly, the rats were placed at the center of an open field box $\left(100 \times 100 \times 40 \mathrm{~cm}^{3}\right)$ with 16 similar small grids drawn at the bottom. After 1 min of adaptation, the number of rearing and crossing that occurred within 5 min was recorded. The chamber was cleaned and wiped with $75 \%$ alcohol after each rat was tested.

\section{Forced Swim Test}

The forced swim test (FST) was performed the day after performing OFT. For this purpose, a cylindrical vessel (measuring $50 \mathrm{~cm}$ in height and $20 \mathrm{~cm}$ in diameter) was used for FST. The vessel was filled with water (up to $30-\mathrm{cm}$ high) at approximately $23{ }^{\circ} \mathrm{C}$. Then, the rats were placed in water, and the time of immobility in $5 \mathrm{~min}$ (with nose above water) was recorded for each rat. Animal immobility was defined as staying still or making small movements in the water to keep the head above the water. The tested rats were then dried and returned to their cage at the end of each test.

\section{Western Blotting}

Samples from the hippocampus tissues $(15 \mathrm{mg}$ ) and $1 \mathrm{~mL}$ of RIPA lysis buffer (Beyotime, Shanghai) were added to a 2-mL EP tube. The solution of the EP tube was homogenized and centrifuged at $12,000 \times g$ at $4{ }^{\circ} \mathrm{C}$ for $10 \mathrm{~min}$, and the supernatant was collected. Samples from HAPI or PC-12 cells were given the following treatment: The treated cells (covering $80 \%$ area of the cell culture dish) were lysed in RIPA and centrifuged to obtain the supernatant. BCA method was used to determine the protein concentration. Proteins were then separated by SDS-PAGE (30 $\mu \mathrm{g} / \mathrm{lane})$ and then transferred onto a PVDF membrane. Skim milk (5\%) dissolved in TBS was added to cover the membranes. After $2 \mathrm{~h}$, the milk solution was discarded, and a primary antibody was incubated with the protein at $4{ }^{\circ} \mathrm{C}$ overnight. Next, the primary antibody was removed, and the membranes were washed with TBST and incubated with secondary antibody for another $1 \mathrm{~h}$. Later, the protein bands were visualized by chemiluminescent immunoassay and quantified by Image $\mathbf{J}$ software. The following antibodies were used in the study: anti-COX-2 antibody (No.360120, Cayman), anti-iNOS antibody (ab178945, Abcam), anti-SOCS3 antibody (ab16030, Abcam), anti-NRF2 antibody (\#33649, Cell Signalling Technology), anti-HO-1 antibody (\#82206, Cell Signalling Technology), anti-EZH2 antibody (ab283270, Abcam), and anti-beta-actin (ab8226, Abcam).

\section{ELISA Assay for IL-1 $\beta$, TNF- $\alpha$, and IL-6}

Whole blood samples of rats were obtained through eyeball extraction and stored at room temperature for $2 \mathrm{~h}$, followed by centrifugation at $1500 \times g$ for $10 \mathrm{~min}$ to collect the serum. The levels of IL- $1 \beta$, TNF- $\alpha$, or IL- 6 were detected by using the enzyme-linked immunoassay (ELISA) assay kit (CUSABIO, Wuhan) with serum or hippocampus tissue lysate. Briefly, 50 $\mu \mathrm{L}$ of the samples or standards were added to each well, followed by the addition of a $50-\mu \mathrm{L}$ of antibody cocktail that was incubated at $37^{\circ} \mathrm{C}$ for $1 \mathrm{~h}$. At the end of the incubation, the mixture was discarded, and the plate was washed with PBS. The TMB development solution was added for $10 \mathrm{~min}$. Finally, $100 \mu \mathrm{L}$ of the stop solution was added, and the optical density (OD) at $450 \mathrm{~nm}$ was recorded with a luminometer.

\section{ATP Production Assay}

The ATP Assay Kit (Biotime, Shanghai) was used to test the production of ATP in the hippocampus tissues. For this purpose, the standard curve and the ATP working solution were prepared as suggested by the manufacturer's instructions. A $50 \mu \mathrm{L}$ of harvested hippocampus tissue lysate was added to each testing well, followed by the addition of 100 $\mu \mathrm{L}$ of ATP working solution to react for 3-5 min, followed by recording the OD value and calculation of the ATP concentration concerning the standard curve.

\section{Observation of Mitochondrial Morphology}

The hippocampal tissues $\left(<1 \mathrm{~mm}^{3}\right)$ were fixed with $2.5 \%$ glutaraldehyde for $2 \mathrm{~h}$, followed by washing with BPS and fixation with $1 \%$ osmic acid for $2 \mathrm{~h}$ and a final rinse with PBS. Gradually, the tissue was dehydrated with different concentrations of ethyl alcohol and acetone and then embedded with acetone solution overnight. After fixation, the tissue was cut into $50-\mathrm{nm}$ slices and stained with $3 \%$ uranium acetate and lead citrate, and the mitochondrial morphology was then observed under a transmission electron microscope.

\section{Immunofluorescence Assay}

The paraffin sections of the hippocampal tissues were subjected to an immunofluorescence (IF) assay. Briefly, the sections were washed with TBS containing $0.025 \%$ Triton X-100 and then incubated with $10 \%$ FBS for $2 \mathrm{~h}$ at room temperature, followed by incubation with anti-Iba1 antibody $(1: 100$, Abcam, ab178847) at $4{ }^{\circ} \mathrm{C}$ overnight. The sections were then 
incubated with a fluorescent secondary antibody and DAPI in the dark and photographed under a fluorescence microscope.

\section{Mitochondrial DNA Isolation Procedure}

Mitochondrial DNA (mtDNA) was integrity isolated using the mtDNA Isolation Kit (BioVision) according to the manufacturer's instruction. Briefly, homogenized hippocampus tissue samples were centrifuged at $1200 \times g$ for $10 \mathrm{~min}$ at $4{ }^{\circ} \mathrm{C}$, and the supernatant was centrifuged at $10,000 \times g$ for $15 \mathrm{~min}$ at $4{ }^{\circ} \mathrm{C}$, after which the mitochondria were isolated in the precipitation. Enzyme B Mix was added to the mitochondrial extract and incubated at $50{ }^{\circ} \mathrm{C}$ in a water bath for $60 \mathrm{~min}$ until the solution became clear. The DNA pellet was finally washed with ethanol to obtain mtDNA for the subsequent qPCR test.

\section{Mitochondrial ROS Production Assay}

The MitoSOX Red Mitochondrial Superoxide Indicator Kit (Invitrogen) was used to examine the mitochondrial ROS production. For this, a $5-\mu \mathrm{M}$ of the working solution was prepared and added to treat the PC-12 cells for $10 \mathrm{~min}$ at $37{ }^{\circ} \mathrm{C}$ away from direct light. Then, the stained cells were mounted in a warm buffer for subsequent imaging.

\section{Mitochondrial Membrane Potential Assay}

To determine the mitochondrial membrane potential, the JC-1 stain (JC-1 kit, Invitrogen) was diluted to $2 \mu \mathrm{g} / \mathrm{mL}$ concentration first. The treated PC-12 cells were harvested and washed with PBS and then incubated with the JC-1 stain working solution. The excess dye was washed off after $15 \mathrm{~min}$, and the fluorescence intensity of the red and green color was observed under a fluorescence microscope.

\section{Quantitative Real-Time PCR}

Total RNA was extracted from the tissue homogenate or cells using a TRIzol reagent (Invitrogen), chloroform, isopropanol, and $75 \%$ ethanol. After RNA purification, DEPC water was used to dissolve the extract to determine the concentration. For reserve transcription to synthesize cDNA, $1000 \mathrm{ng}$ of the RNA was added to Takara RT reagent (Japan) and allowed to react at $37{ }^{\circ} \mathrm{C}$ for $15 \mathrm{~min}$ for 3 cycles and then at $85{ }^{\circ} \mathrm{C}$ for $5 \mathrm{~s}$. Finally, $2 \mu \mathrm{L}$ of the cDNA solution was applied for qRT-PCR using the SYBR Premix Kit (Takara) according to the manufacturer protocol. The reaction system was carried out at $95^{\circ} \mathrm{C}$ for $30 \mathrm{~s}, 95^{\circ} \mathrm{C}$ for $5 \mathrm{~s}$, and $60^{\circ} \mathrm{C}$ for $34 \mathrm{~s}$ for 40 repeats. The quantification of the target RNA level was calculated through the $2^{-\Delta \Delta C T}$ method. The primer sequences were as follows $\left(5^{\prime}-3^{\prime}\right)$ : GAS5 forward GAGTGGGTGGGAAGT
CTGAA, reverse GAGTGGGTGGGAAGTCTGAA; SOCS3 forward GCCTCAAGACCTTCAGCTCCAAG, reverse CGG TTACGGCACTCCAGTAGAATC; NRF2 forward GCCTTC CTCTGCTGCCATTAGTC, reverse TGCCTTCAGTGTGCT TCTGGTTG; GAPDH forward GTGGACCTCATGGCC TACAT, reverse TGTGAGGGAGATGCTCAGTG.

\section{RNA-Binding Protein Immunoprecipitation}

The RIP Immunoprecipitation Kit (Millipore, USA) was used for the RNA-binding protein immunoprecipitation (RIP) experiment. HAPI or PC-12 cells were collected and resuspended in $200 \mu \mathrm{L}$ of the RIP lysis with protease inhibitor and RNase inhibitor and then centrifuged at $14,000 \times g$ at $4{ }^{\circ} \mathrm{C}$ for $10 \mathrm{~min}$, and then, the supernatant was aspirated into another EP tube. In addition, protein $\mathrm{A} / \mathrm{G}$ magnetic beads were washed and resuspended in $100 \mu \mathrm{L}$ of RIP wash buffer, followed by incubation with $5 \mu \mathrm{L}$ of EZH2 antibody (Abcam, ab283270) or $\mathrm{IgG}$ and rotated for $1 \mathrm{~h}$ at room temperature. Then, the EP tubes containing magnetic beads were placed on the magnet to retain the precipitation at the bottom. Next, $900 \mu \mathrm{L}$ of RIP immunoprecipitation buffer and $100 \mu \mathrm{L}$ of the cell lysate were added to the tube for incubation with the bead-antibody complex at $4{ }^{\circ} \mathrm{C}$ for rotation for $3 \mathrm{~h}$. The next day, $150 \mu \mathrm{L}$ of proteinase $\mathrm{K}$ buffer was added to each tube, and the resultant solution was mixed at $55^{\circ} \mathrm{C}$ for $30 \mathrm{~min}$. Furthermore, the tube was transferred to the magnet, and the supernatant was collected and combined with phenol, chloroform, and isoamylol to extract RNA from the immunoprecipitation. Finally, the RNA samples were prepared for qPCR to verify the potential target relationship between the GAS5 and EZH2 proteins.

\section{RNA Pull-Down}

Sense with T7 promoter and antisense of GAS5 were synthesized, and in vitro transcription was subjected using respective primers by mMESSAGE mMACHINE kit (Ambion, USA). RNA size was detected by agarose gel. According to the manufacturer's instruction, the biotin-labeled RNA (Bio-RNA) was prepared using an RNA 3'-end desthiobiotinylation kit (Thermo). The labeled RNA was purified immediately, followed by incubation with $50 \mu \mathrm{L}$ magnetic beads at room temperature for $1 \mathrm{~h}$ rotation. The HAPI or PC-12 cells lysate mixed with $200 \mathrm{U} / \mathrm{mL}$ of RNase inhibitor and incubated with the magnetic beads overnight at $4{ }^{\circ} \mathrm{C}$. Finally, the mixture was replaced with the magnet, and the beads were rinsed with the cell lysis buffer to collect the target protein, followed by western blotting.

\section{Chromatin Immunoprecipitation-qPCR (CHIP-qPCR)}

The HAPI or PC-12 cells were washed with cold PBS and treated with $1 \%$ formaldehyde for $10 \mathrm{~min}$ to fix 
reversible protein-DNA cross-link and then mixed with glycine $(125 \mathrm{mM})$ to quench the excess formaldehyde at room temperature. Subsequently, the cells were scraped and incubated with SDS lysis buffer containing proteinase inhibitor, then sonicated to cleave chromatin into a small fragment. Thus, after centrifugation, the supernatant was incubated with ChIP dilution buffer, $50 \times$ PIC, and magnet beads with EZH2 or H3K27me3 antibody (ab6002, Abcam) or IgG prepared in advance. The next day, the immunoprecipitation complex was washed with the following solutions: low-salt wash buffer, high-salt wash buffer, $\mathrm{LiCl}$, and wash buffer once and with TE buffer twice, after which the complex was eluted with a $10 \%$ SDS and $100 \mathrm{uL} / \mathrm{M} \mathrm{NaHCO}_{3}$ dissolved in a $\mathrm{ddH}_{2} \mathrm{O}$ for overnight. The DNA was harvested using the Gene Elute kit (Merck) according to the protocol and analyzed by the qPCR assay.

\section{Statistical Analysis}

GraphPad Prism 8.0 was used for data analysis. The data were recorded from no less than three independent experiments and expressed as mean \pm SD. Student's $t$ test or oneway ANOVA was applied to compare the statistical difference, and $P \leq 0.05$ was considered to indicate statistical significance.

\section{Results}

\section{Rg1 Ameliorated Depression-Like Behaviours in CRS-Induced Rats}

We first investigated the anti-depressive effect of $\operatorname{Rg} 1$ on CRS-induced rats. An open field test revealed that CRS
Fig. $1 \mathrm{Rg} 1$ ameliorated depression-like behaviours in CRS-induced rats. CRS rats were restricted in an adjustable cylindrical plastic bound pipe fitted to allow them to breathe for $4 \mathrm{~h}$ every day (from 09:00 to 13:00) with no food and water for 28 consecutive days. A group of rats (control) was not subjected to any treatment other than restraining food and water supply during 09:00-13:00. Rg1 (20 mg/kg/ day) or escitalopram (1 mg/kg/ day) was administered to the rats via the intragastric route $1 \mathrm{~h}$ after CRS every day. After $24 \mathrm{~h}$ accommodation, behaviours tests were performed. A and $\mathbf{B}$ Rearing and crossing counts in open field test. C Sucrose preference test. D Immobility time in forced swim test. $* P<0.05$; ** $P<0.01$; *** $P<0.001$
A

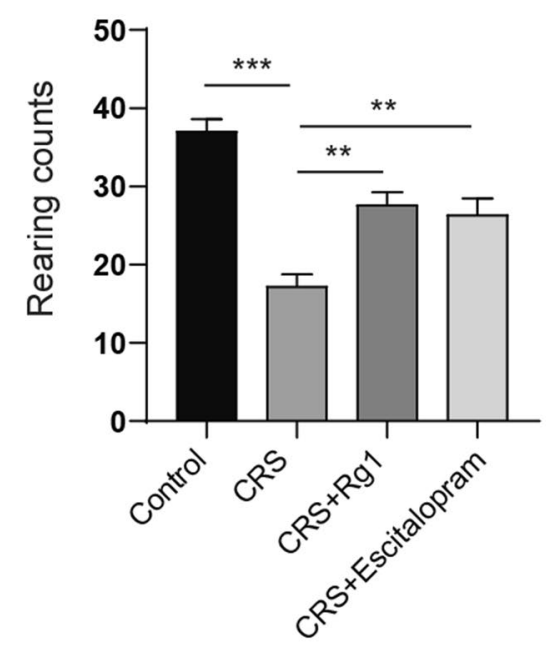

C

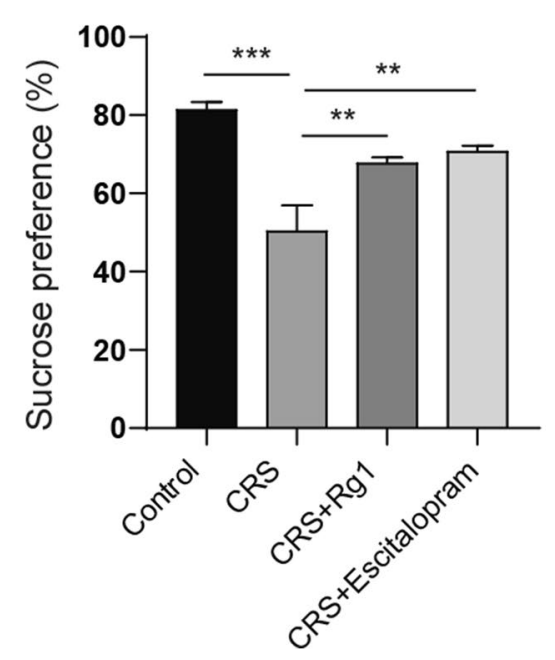

B
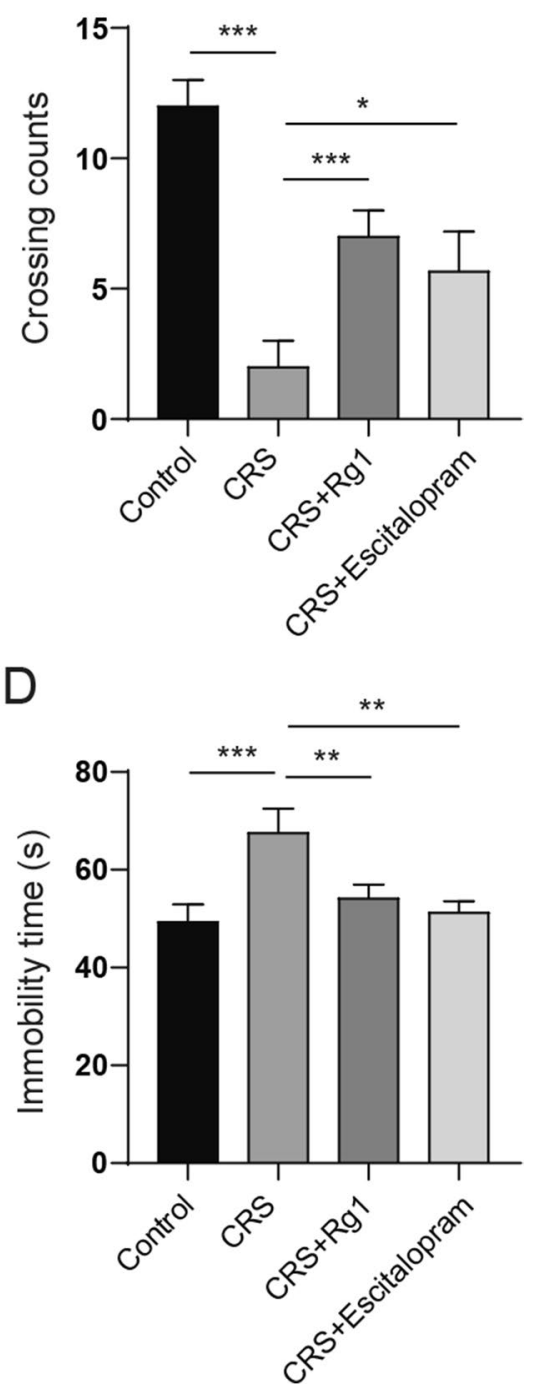
A

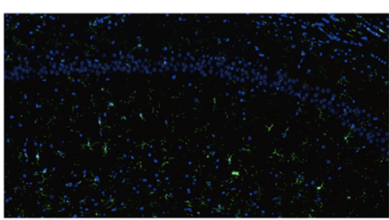

Control

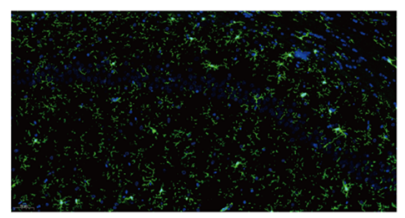

CRS

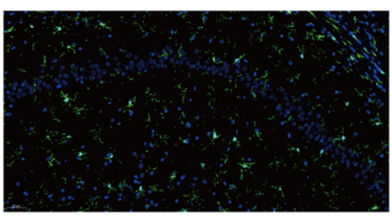

CRS+Rg1

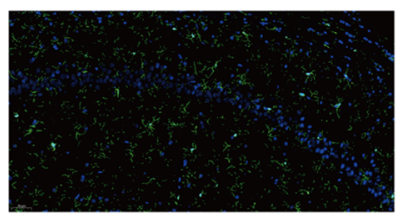

CRS+Escitalopram
B

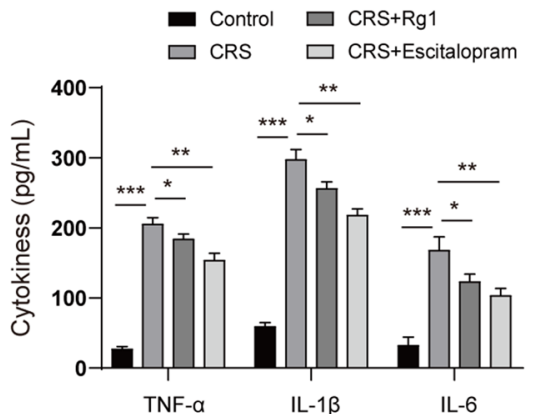

C

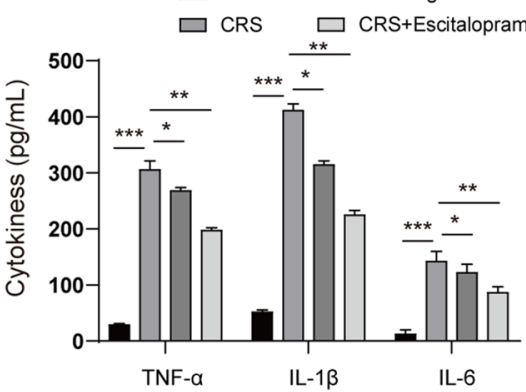

$\mathrm{D}$

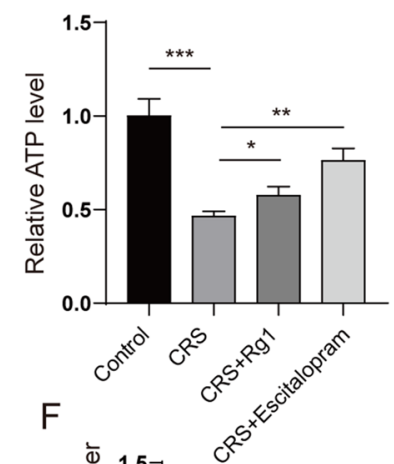

$E$

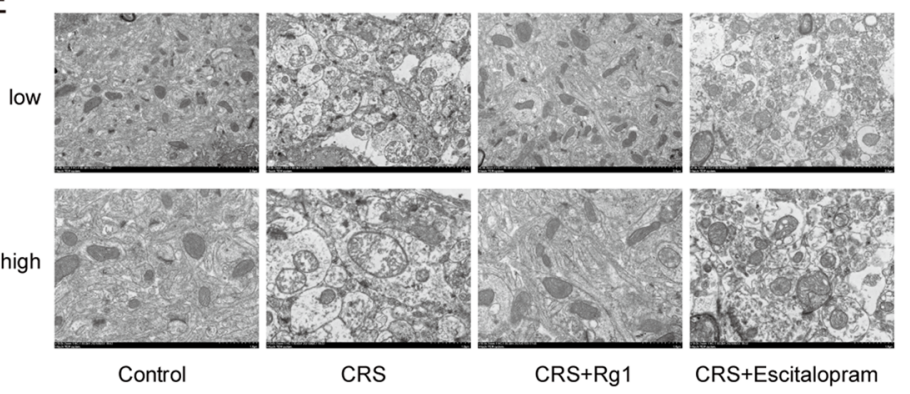

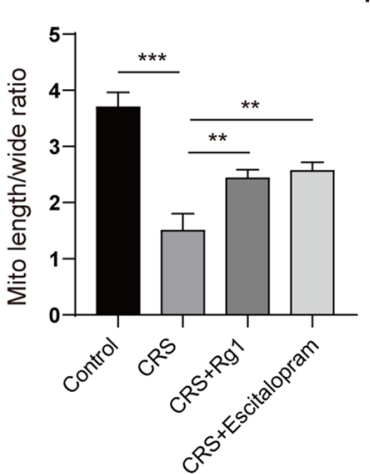

$\mathrm{H}$

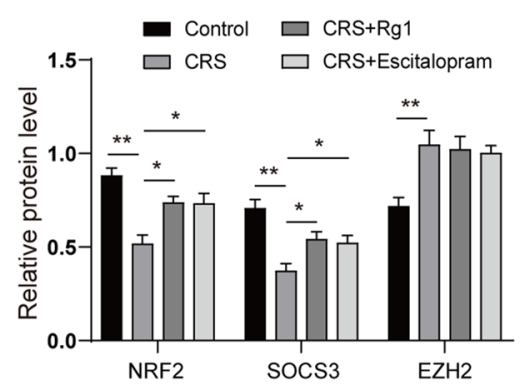

G

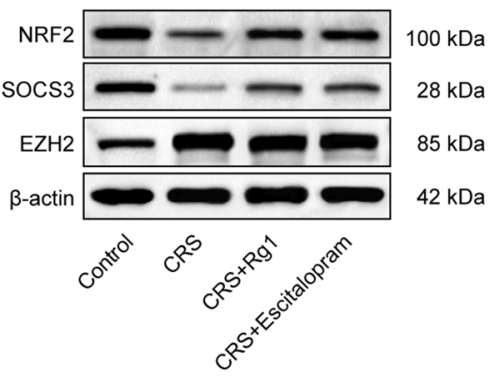

Fig. 2 Rg1 abrogated microglial activation and attenuated mitochondrial dysfunction in CRS-induced rats. CRS rats were restricted in an adjustable cylindrical plastic bound pipe fitted to allow them to breathe for $4 \mathrm{~h}$ every day (from 09:00 to 13:00) with no food and water for 28 consecutive days. A group of rats (control) was not subjected to any treatment other than restraining food and water supply during 09:00-13:00. Rg1 (20 mg/kg/day) or escitalopram (1 mg/ $\mathrm{kg}$ /day) was administered to the rats via the intragastric route $1 \mathrm{~h}$ after CRS every day. Hippocampus and serum were collected. A
IBA-1 positive cells in the hippocampus were detected by immunofluorescence. B and C Pro-inflammatory cytokines (TNF- $\alpha$, IL-1 $\beta$, and IL-6) in the serum (B) and hippocampus (C) were detected by ELISA. D ATP level in hippocampus was detected. E The morphology of mitochondria in hippocampus was observed by TEM. F The copy number of mitochondrial DNA (mtDNA) in hippocampus. G The protein levels of SOCS3, NRF2, and EZH2 in hippocampus were detected by western blots. $\mathbf{H}$ The level of GAS5 was detected by qPCR. $* P<0.05 ; * * P<0.01 ; * * * P<0.001$ 
treatment notably reduced the rearing and crossing counts. However, the injection of $\mathrm{Rg} 1$ or escitalopram (positive control) restored the rearing and crossing counts (Fig. 1A and Fig. 1B). Sucrose preference test revealed that Rg1 and escitalopram recovered CRS reduced sucrose consumption (Fig. 1C). Additionally, the forced swim test illustrated that CRS increased the immobility time of rats, whereas the injection of $\mathrm{Rg} 1$ or escitalopram reduced it (Fig. 1D). These results confirmed that $\mathrm{Rg} 1$ treatment alleviated CRS-induced depression-like behaviours in CRS rats.

\section{Rg1 Abrogated Activation of Microglial and Attenuated Mitochondrial Dysfunction in CRS-Induced Rats}

Overactivation of microglia is involved in the pathogenesis of depression. We next investigated whether $\operatorname{Rg} 1$ affected microglial activation in CRS-induced rats. As shown in Fig. 2A, CRS increased IBA-1 positive cells (a microglia marker) in the hippocampus, whereas $\operatorname{Rg} 1$ or escitalopram treatment substantially decreased the IBA-1 positive cells. Moreover, we also discovered that the levels of pro-inflammatory cytokines (TNF- $\alpha$, IL-1 $\beta$, and IL-6) in the serum and hippocampus were dramatically elevated by CRS treatment. However, $\operatorname{Rg} 1$ or escitalopram reduced the levels of these pro-inflammatory cytokines (Fig. 2B and Fig. 2C).

Fig. 3 GAS knockdown ameliorated depression-like behaviours in CRS-induced rats. In vivo GAS5 knockdown was achieved by injecting lentivirus-packaged GAS5 shRNA (Lenti-shGAS5) into the lateral ventricle at the following coordinates: $0.3 \mathrm{~mm}$ behind the bregma and $1.0 \mathrm{~mm}$ lateral from the sagittal midline, at a depth of $2.2 \mathrm{~mm}$ from the skull surface on the last day of adaptation by using a Nanoject II (Drummond) system at a rate of $0.1 \mu \mathrm{l}$ per min.. Forty-eight hours after in vivo injection, the rats were subjected to CRS stimulation for 28 days. After $24 \mathrm{~h}$ accommodation, behaviours tests were performed. A The GAS5 level in hippocampus was detected by qPCR. $\mathbf{B}$ and $\mathbf{C}$ Rearing and crossing counts in the open field test. D Sucrose preference test. $\mathbf{E}$ Immobility time in the forced swim test. $* P<0.05$; $* * P<0.01 ; * * * P<0.001$
A

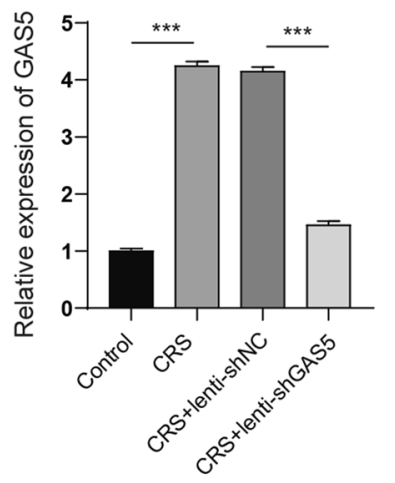

D

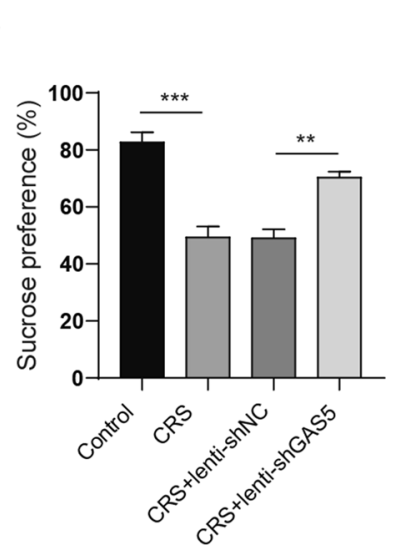

Mitochondrial dysfunction was also commonly observed in depression. In the present study, we found that the ATP level notably decreased in the CRS-induced group. Rg1 or escitalopram partly restored the ATP level (Fig. 2D).

Additionally, the transmission electron microscopy (TEM) analysis of mitochondrial morphology revealed mitochondrial injury and a decrease in the length-width ratio of mitochondria in the CRS group. However, $\operatorname{Rg} 1$ or escitalopram alleviated mitochondrial injury and restored the length-width ratio of mitochondria (Fig. 2E). Moreover, CRS decreased the copy number of mitochondrial DNA (mtDNA), whereas Rg1 or escitalopram recovered the copy number of mtDNA (Fig. 2F). To investigate Rg1 regulation of microglial activation and mitochondrial function in depression, we analyzed the expression of several proteins that might be involved in the progress of inflammatory response and oxidative stress. As shown in Fig. 2G, the results illustrated that $\mathrm{Rg} 1$ and escitalopram restored the expressions of SOCS3 and NRF2, which were reduced in CRS-treated mice. SOCS3 and NRF2 were negatively regulated by EZH2-mediated epigenetic suppression [28, 29]. We also tested whether $\operatorname{Rg} 1$ influences the expression of EZH2. The results indicated that EZH2 was elevated in the CRS rat model. However, $\operatorname{Rg} 1$ treatment failed to exhibit a significant change in the expression of EZH2. GAS5 was reported as a lncRNA that enhanced the suppressive function

B

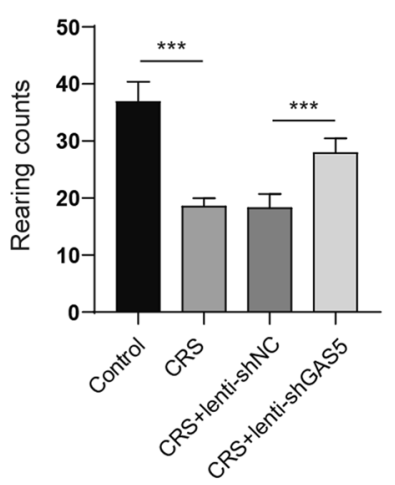

E

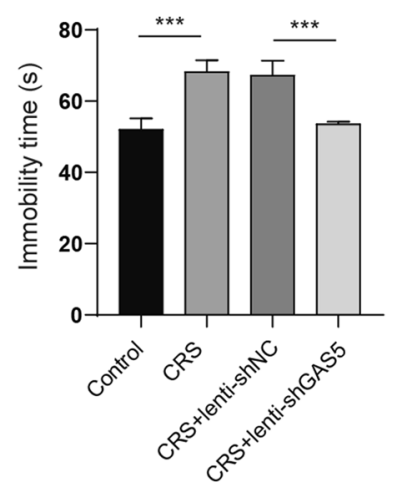

C

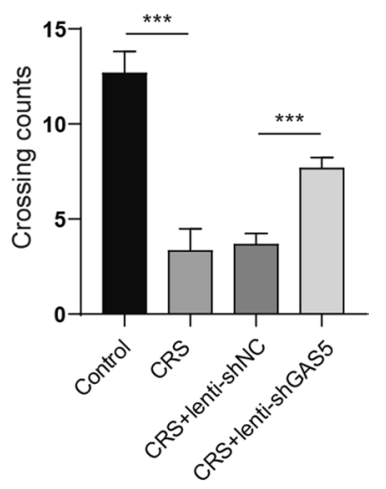


A

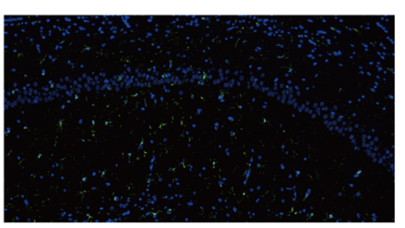

Control

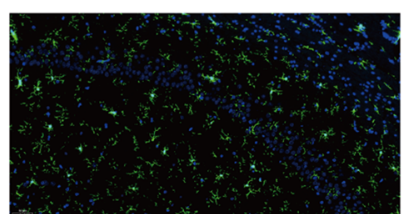

CRS

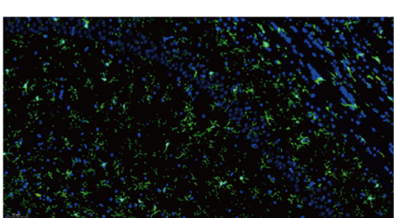

CRS+lenti-shNC

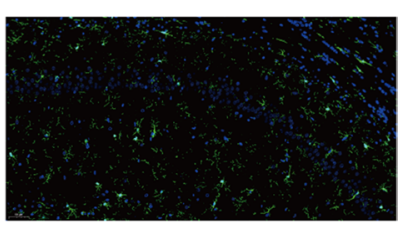

CRS+lenti-shGAS5
B

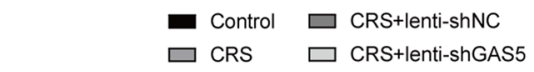

C $\square$ Control $\square$ CRS+lenti-shNC
$\square$ CRS $\square$ CRS+lenti-shGAS5

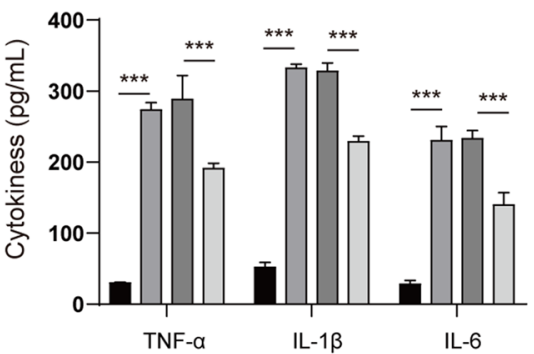

D

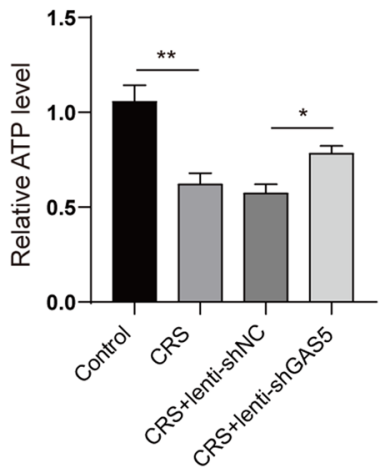

E

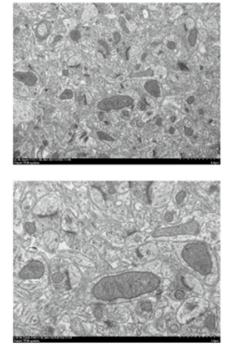

Control

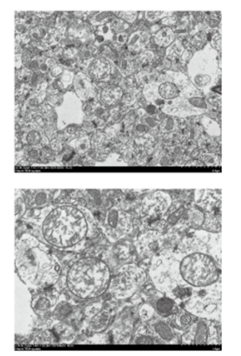

CRS
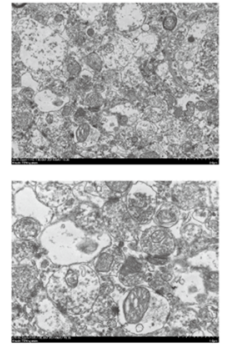

CRS+lenti-shNC

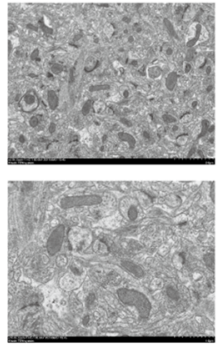

CRS+lenti-shGAS5

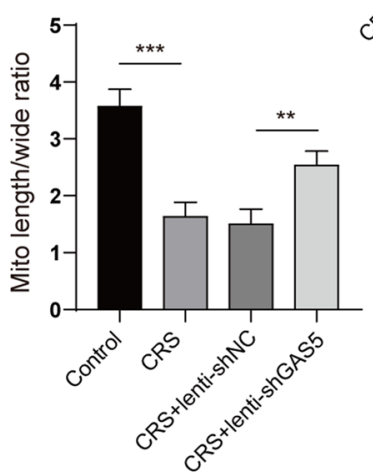

F

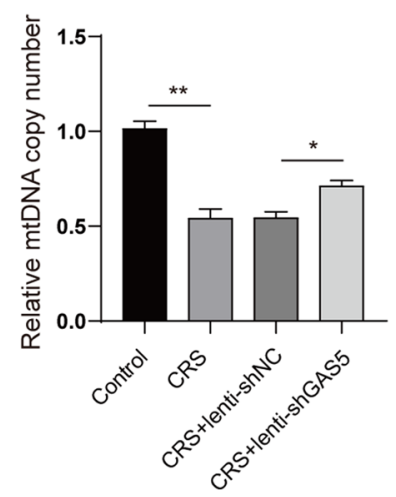

G

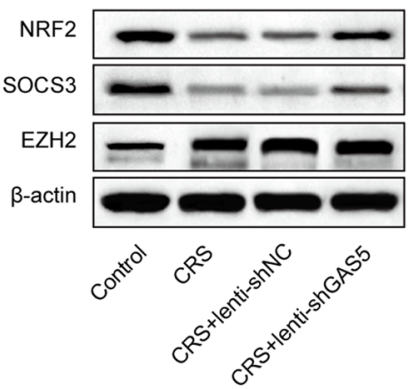

$100 \mathrm{kDa}$

$28 \mathrm{kDa}$

$85 \mathrm{kDa}$

$42 \mathrm{kDa}$

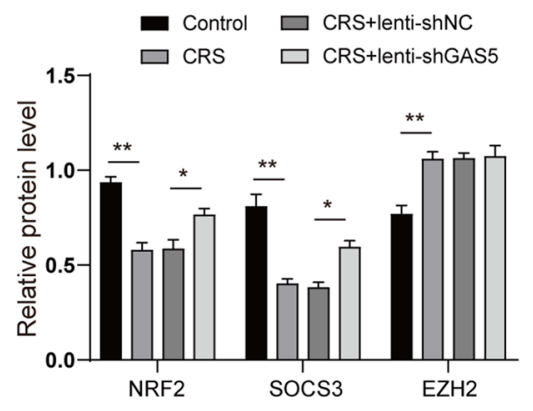

Fig. 4 GAS5 knockdown suppressed microglial activation and attenuated mitochondrial dysfunction in CRS-induced rats. In vivo GAS5 knockdown was achieved by injecting lentivirus-packaged GAS5 shRNA (Lenti-shGAS5) into the lateral ventricle at the following coordinates: $0.3 \mathrm{~mm}$ behind the bregma and $1.0 \mathrm{~mm}$ lateral from the sagittal midline, at a depth of $2.2 \mathrm{~mm}$ from the skull surface on the last day of adaptation by using a Nanoject II (Drummond) system at a rate of $0.1 \mu \mathrm{l}$ per min. Forty-eight hours after in vivo injection, the rats were subjected to CRS stimulation for 28 days. Hippocampus and serum were collected. A IBA-1 positive cells in the hippocampus were detected by immunofluorescence. $\mathbf{B}$ and $\mathbf{C}$ The levels of pro-inflammatory cytokines (TNF- $\alpha$, IL- $1 \beta$, and IL-6) in the serum (B) and hippocampus (C) were detected by ELISA. D ATP level in hippocampus was detected. E The morphology of mitochondria in hippocampus was observed by TEM. F The copy number of mitochondrial DNA (mtDNA) in hippocampus. G The protein levels of SOCS3, NRF2, and EZH2 in hippocampus were detected by western blots. $* P<0.05 ; * * P<0.01 ; * * * P<0.001$ 
A

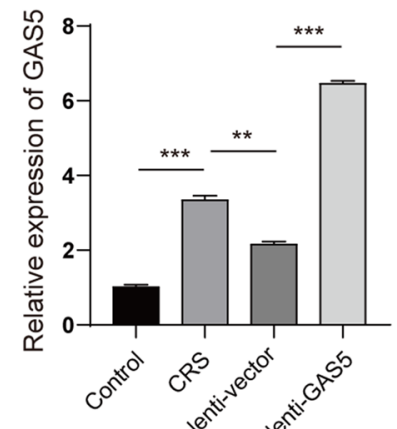

E

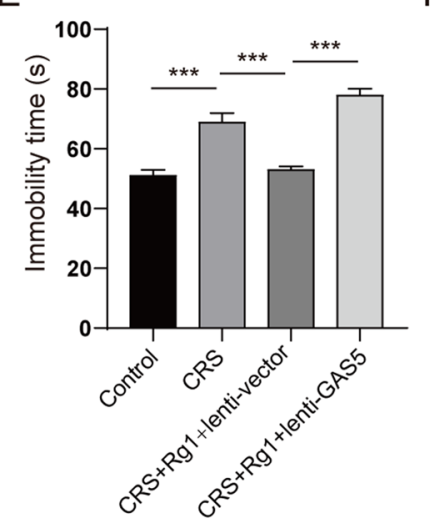

B

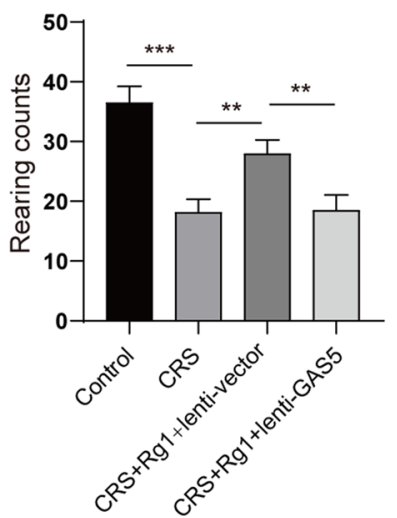

F

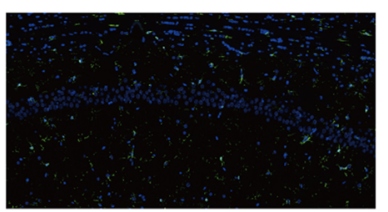

Control

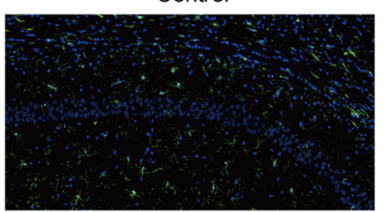

CRS+Rg1+lenti-vector

$\mathrm{H}$

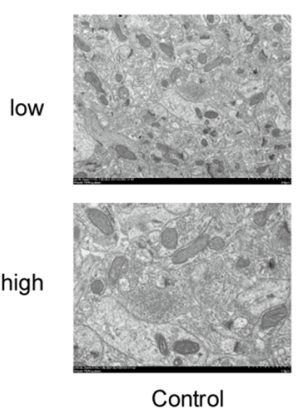

I
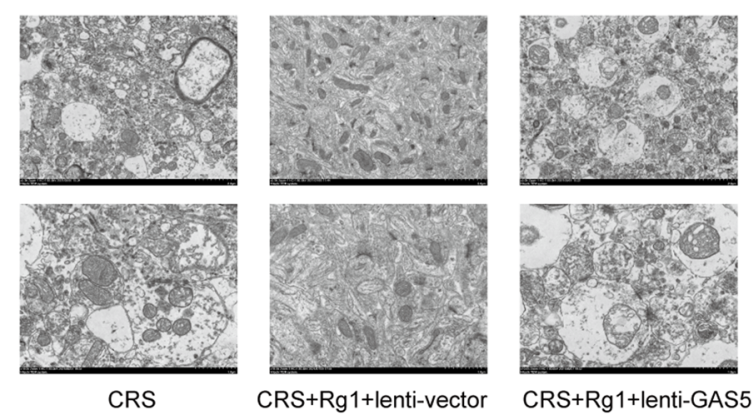

C
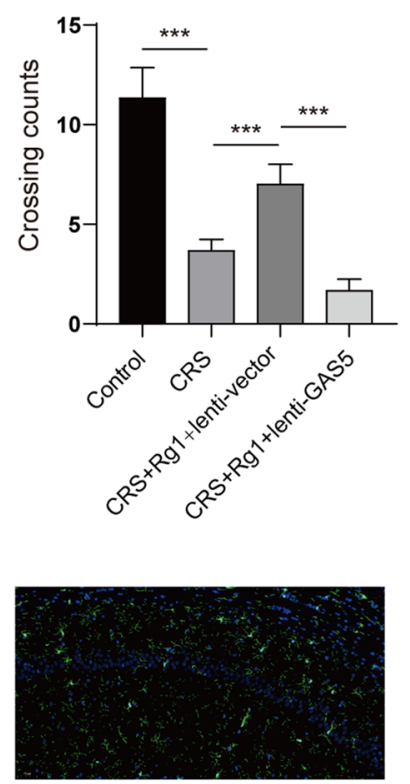

CRS

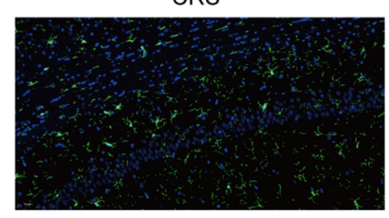

CRS+Rg1+lenti-GAS5
D

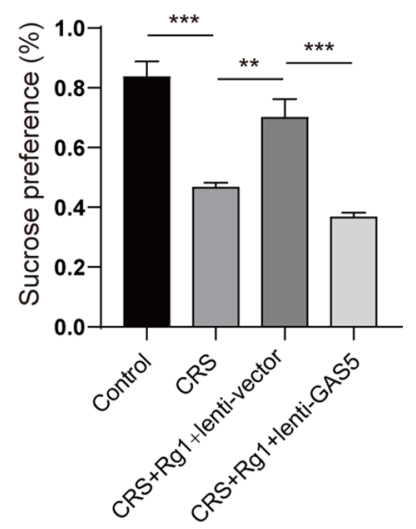

G

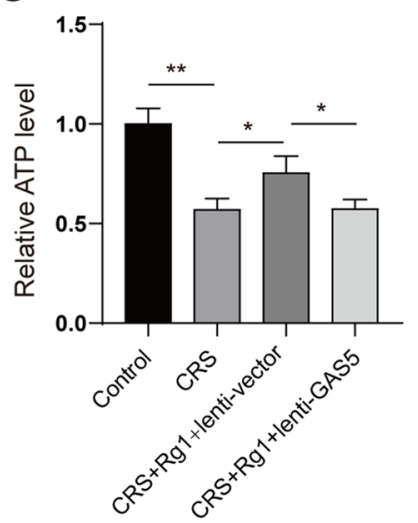

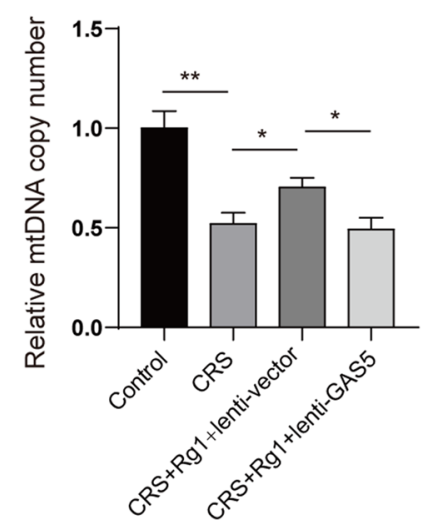

$$
\text { J }
$$
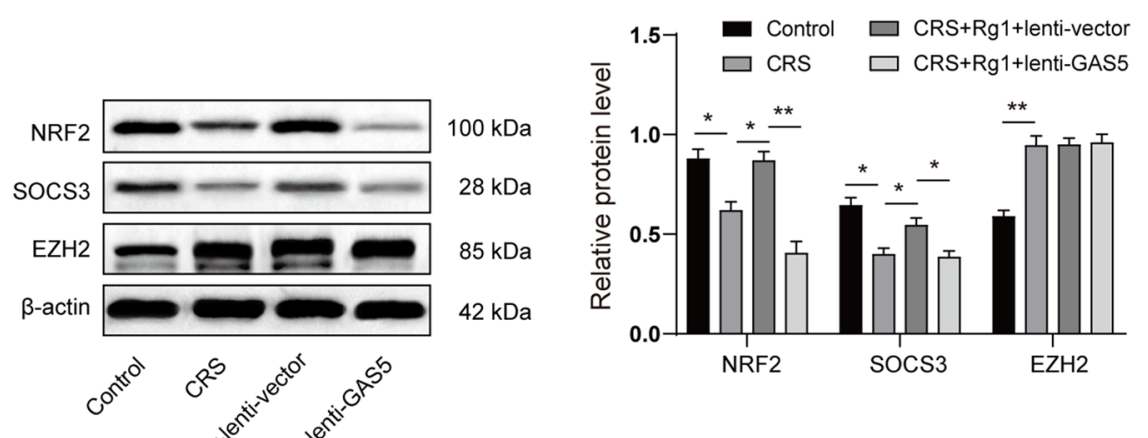
4Fig. $5 \mathrm{Rg} 1$ exerted a protective effect on the rat CRS-induced depression model by downregulating GAS5. In vivo GAS5 knockdown was achieved by injecting lentivirus-packaged GAS5 shRNA (Lenti-shGAS5) into the lateral ventricle at the following coordinates: $0.3 \mathrm{~mm}$ behind the bregma and $1.0 \mathrm{~mm}$ lateral from the sagittal midline, at a depth of $2.2 \mathrm{~mm}$ from the skull surface on the last day of adaptation by using a Nanoject II (Drummond) system at a rate of $0.1 \mu \mathrm{l}$ per min. Forty-eight hours after in vivo injection, rats were subjected to CRS stimulation for 28 days and $\operatorname{Rg} 1(20 \mathrm{mg} / \mathrm{kg})$ treatment every day $1 \mathrm{~h}$ after CRS stimulation. $24 \mathrm{~h}$ after modeling, behaviour tests were performed. A The level of GAS5 in hippocampus was detected by qPCR. B and $\mathbf{C}$ Rearing and crossing counts in the open field test. D Sucrose preference test. E Immobility time in the forced swim test. F IBA-1 positive cells in the hippocampus were detected by immunofluorescence. G ATP level in hippocampus was detected. $\mathbf{H}$ The morphology of mitochondria in hippocampus was observed by TEM. I The copy number of mitochondrial DNA (mtDNA) in hippocampus. J The protein levels of SOCS3, NRF2, and EZH 2 in hippocampus were detected by western blots. $* P<0.05$; $* * P<0.01 ; * * * P<0.001$

of EZH2 on gene transcription [30]. Hence, we also detected the expression of GAS5. Interestingly, we observed that Rg1 treatment notably decreased GAS5 expression induced by CRS (Fig. 2H). Collectively, these results revealed that Rg1 abrogated microglial activation and attenuated mitochondrial dysfunction in CRS-induced rats.

\section{GAS Knockdown Ameliorated Depression-Like Behaviours in CRS-Induced Rats}

We next investigated the anti-depressive effect of GAS5 knockdown on CRS rats. In vivo GAS5 knockdown was achieved by injecting lentivirus-packaged GAS5 short hairpin RNA (shRNA) (Lenti-shGAS5) into the rat brain. qPCR revealed that CRS notably elevated the expression of GAS5, whereas the injection of Ad-shGAS5 significantly repressed the expression of GAS5 in the hippocampus (Fig. 3A). The open field test illustrated that CRS reduced the rearing and crossing counts, whereas the injection of Ad-shGAS5 restored the number of rearing and crossing counts (Fig. 3B and Fig. 3C). Sucrose preference test revealed that GAS5 knockdown recovered the sucrose consumption reduced by CRS (Fig. 3D). Moreover, the forced swim test showed that CRS increased the immobility time of rats, whereas GAS5 knockdown reduced the immobility time (Fig. 3E). These results confirmed that GAS5 knockdown alleviated CRSinduced depression-like behaviours in rats.

\section{GAS5 Knockdown Suppressed Microglial Activation and Attenuated Mitochondrial Dysfunction in CRS-Induced Rats}

We next investigated that whether GAS5 knockdown affected microglial activation and mitochondria dysfunction in CRSinduced rats. As shown in Fig. 4A, CRS increased IBA-1 positive cells in the hippocampus, whereas GAS5 knockdown decreased IBA-1 positive cells. Moreover, the levels of proinflammatory cytokines, including TNF- $\alpha$, IL- $1 \beta$, and IL-6, in the serum and hippocampus were dramatically elevated by CRS treatment, whereas GAS5 knockdown decreased the level of the pro-inflammatory cytokines (Fig. 4B and Fig. 4C). In terms of mitochondrial dysfunction, the ATP level notably reduced in the CRS-induced group, which was restored by GAS5 knockdown (Fig. 4D). Additionally, TEM analysis revealed the mitochondrial injury and a decrease in the mitochondrial length-width ratio in the CRS group. However, GAS5 knockdown ameliorated mitochondrial injury and recovered the length-width ratio of mitochondria (Fig. 4E). Similarly, our results also suggested that GAS5 knockdown restored the copy number of mtDNA decreased by CRS treatment (Fig. 4F). We also tested whether GAS5 regulated the expression of NRF2, SOCS3, and EZH2 in the CRS model. As shown in Fig. 4G, the results illustrated that GAS5 restored the expressions of SOCS3 and NRF2, which were suppressed in CRS-treated mice. However, the GAS5 knockdown failed to reveal a significant influence on the expression of EZH2. Collectively, these results demonstrated that GAS5 knockdown suppressed microglial activation and attenuated mitochondrial dysfunction in CRS-induced rats.

\section{Rg1 Exerted a Protective Effect on the Rat CRS-Induced Depression Model Downregulating GAS5}

To investigate whether GAS5 mediated the protective effect of Rg1 in depression, we injected lentivirus-packaged GAS5 overexpressing plasmid (Lenti-GAS5) into the rat brain before $\operatorname{Rg} 1$ treatment and modeling. As shown in Fig. 5A, the overexpression of GAS5 substantially elevated the Rg1reduced expression of GAS5 in the hippocampus. Behavioural experiments suggested that $\mathrm{Rg} 1$ alleviated depressionlike behaviours, as indicated by increased rearing counts, crossing counts, sucrose consumption, and decreased immobility time. However, the overexpression of GAS5 partly compromised the protective effect of $\mathrm{Rg} 1$ on depression-like behaviours (Fig. 5B to Fig. 5E). Moreover, GAS5 reversed the effect of $\mathrm{Rg} 1$ on microglial activation and mitochondrial dysfunction. Immunofluorescence revealed that $\operatorname{Rg} 1$ reduced IBA-1 positive cells, whereas tGAS5 overexpression reversed this result (Fig. 5F). Additionally, the overexpression of GAS5 compromised the Rg1-induced increase in the ATP level, length-width ratio, and mtDNA copy number (Fig. 5G to I). Rg1 consistently increased the expression of SOCS3 and NRF2 in the depression model, whereas the GAS5 overexpression compromised this effect, as indicated by a notable decrease in the expression of SOCS3 and NRF2 (Fig. 5J). Overall, our results indicated that GAS5 mediated the protective effect of $\operatorname{Rg} 1$ in depression. 


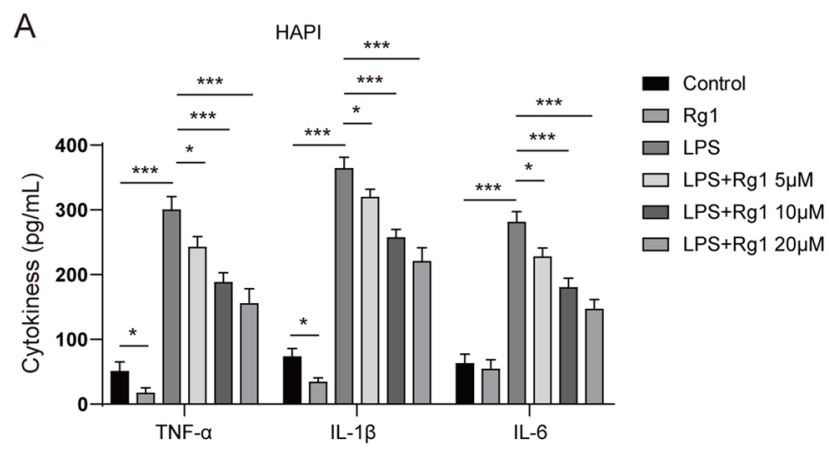

B
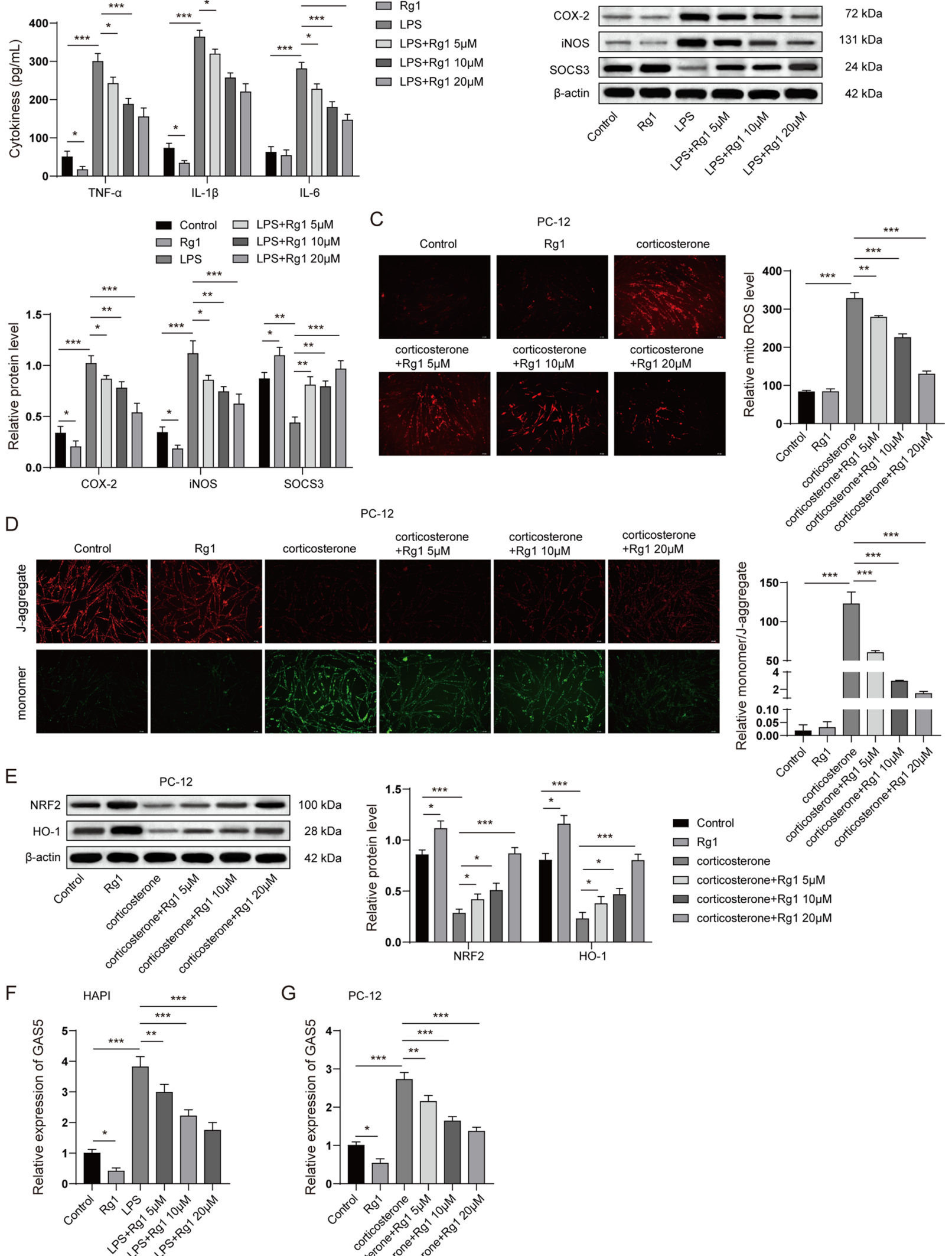

G

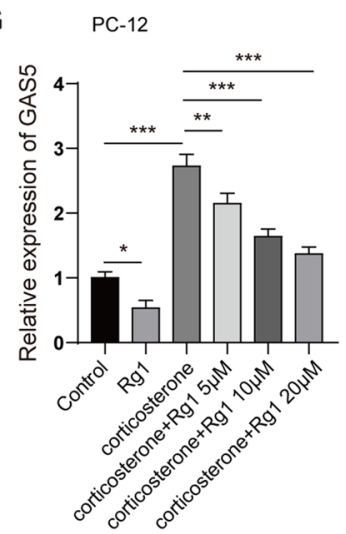


4Fig. 6 Rg1 alleviated microglial activation and neuronal mitochondria dysfunction in vitro. $\operatorname{Rg} 1$ alleviated microglial activation and neuronal mitochondria dysfunction in vitro. A and B Rat microglia cell line HAPI was stimulated with LPS $(1 \mu \mathrm{M})$ or solution control for $2 \mathrm{~h}$, followed by co-incubation with $\operatorname{Rg} 1(5 \mu \mathrm{M}, 10 \mu \mathrm{M}, 20$ $\mu \mathrm{M})$ for a total $24 \mathrm{~h}$. A The release of pro-inflammatory cytokines in supernatant of HAPI cells was detected by ELISA. B The protein level of COX-2, iNOS, and SOCS3 in HAPI cells was detected by western blots. $\mathbf{C}$ to $\mathbf{E}$ PC-12 cells were stimulated with corticosterone $(400 \mu \mathrm{M})$ or solution control for $2 \mathrm{~h}$, followed by co-incubation with $\operatorname{Rg} 1(5 \mu \mathrm{M}, 10 \mu \mathrm{M}, 20 \mu \mathrm{M})$ for a total $24 \mathrm{~h}$. C Mitochondrial ROS in PC-12 cells was detected by the mitoSOX kit. D Mitochondrial membrane potential in PC-12 cells was detected by the JC-1 assay. E The expression of NRF2 and HO-1 in PC-12 cells was detected by western blots. F Rat microglia cell line HAPI was stimulated with LPS (1 $\mu \mathrm{M})$ or solution control for $2 \mathrm{~h}$, followed by co-incubation with $\mathrm{Rg} 1$ $(5 \mu \mathrm{M}, 10 \mu \mathrm{M}, 20 \mu \mathrm{M})$ for a total $24 \mathrm{~h}$. The expression of GAS5 was detected by qRT-PCR. G PC-12 cells were stimulated with corticosterone $(400 \mu \mathrm{M})$ or solution control for $2 \mathrm{~h}$, followed by co-incubation with $\operatorname{Rg} 1(5 \mu \mathrm{M}, 10 \mu \mathrm{M}, 20 \mu \mathrm{M})$ for a total $24 \mathrm{~h}$. The expression of GAS5 was detected by qRT-PCR. $* P<0.05 ; * * P<0.01 ; * * * P<$ 0.001

\section{Rg1 Alleviated Microglial Activation and Neuronal Mitochondria Dysfunction In Vitro}

To validate the findings of our in vivo study, we next investigated the effect of $\operatorname{Rg} 1(5 \mu \mathrm{M}, 10 \mu \mathrm{M}, 20 \mu \mathrm{M})$ on microglial activation and neuronal mitochondrial dysfunction in vitro. Rat microglia cell line HAPI was stimulated with LPS to mimic microglial activation in vitro. Enzyme-linked immunoassay revealed that LPS dramatically promoted the release of pro-inflammatory cytokines, whereas $\mathrm{Rg} 1$ treatment suppressed the pro-inflammatory cytokine levels in the cell culture supernatant (Fig. 6A). Interestingly, $\operatorname{Rg} 1$ treatment alone also decreased the level of TNF- $\alpha$ and IL- $1 \beta$ while showing no significant effect on IL6. Moreover, LPS stimulation significantly elevated the expression of the pro-inflammatory proteins COX-2 and iNOS and decreased the expression of SOCS3 in HAPI cells. Consistent with our expectations, Rg1 treatment reduced COX-2 and iNOS expressions and restored the expression of SOCS3 in LPS-stimulated HAPI cells. $\mathrm{Rg} 1$ treatment alone also led to a decrease of the basal expression of COX-2 and iNOS and upregulated SOCS3 (Fig. 6B). PC-12 cells were subjected to corticosterone stimulation to mimic in vitro mitochondrial dysfunction in depression. As shown in Fig. 6C, corticosterone stimulation notably increased the level of mitochondrial ROS in PC-12 cells, whereas $\mathrm{Rg} 1$ treatment sharply reduced the level of mitochondrial ROS. Mitochondrial membrane potential was detected using the JC- 1 assay. The results suggested that corticosterone stimulation lowered the mitochondrial membrane potential (MtMP) $(\Delta \psi \mathrm{m})$, as indicated by the JC-1 aggregate-monomer ratio. Interestingly, the $\operatorname{Rg} 1$ treatment substantially restored the MtMP (Fig. 6D). $\operatorname{Rg} 1$ treatment alone did not show a notable influence on the basal ROS and MMP (Fig. 6C and 6D). Moreover, the expression of NRF2 and its downstream protein HO-1 were suppressed in the presence of corticosterone, which was recovered by $\operatorname{Rg} 1$ treatment. $\operatorname{Rg} 1$ treatment alone also led to an increase of the basal expression of NRF2 and HO-1 (Fig. 6E). Additionally, we validated that LPS and corticosterone significantly upregulated the level of GAS5 while Rg1 treatment led to a notable decrease of GAS5. Rg1 treatment alone also attenuated the basal expression of GAS5 (Fig. 6F and 6G). Collectively, we demonstrated that $\mathrm{Rg} 1$ alleviated microglial activation and neuronal mitochondrial dysfunction in vitro.

\section{GAS5 Knockdown Alleviated Microglial Activation and Neuronal Mitochondrial Dysfunction In Vitro}

We next investigated the effect of GAS5 knockdown on microglial activation and neuronal mitochondrial dysfunction in vitro. qPCR revealed that GAS5 shRNA sharply diminished the expression of GAS5 upon LSP stimulation (Fig. 7A). Moreover, the results revealed that LPS notably enhanced the release of pro-inflammatory cytokines in HAPI cells, whereas GAS5 knockdown suppressed the level of pro-inflammatory cytokines in the cell culture supernatant (Fig. 7B). LPS stimulation also elevated the expression of COX-2 and iNOS and reduced SOCS3 expression. Interestingly, GAS5 knockdown reduced COX-2 and iNOS expressions but restored the expression of SOCS3 (Fig. 7C). In terms of mitochondrial dysfunction, as shown in Fig. 7D and E, corticosterone stimulation substantially increased the level of GAS5 and mitochondrial ROS in PC-12 cells, whereas GAS5 knockdown significantly attenuated the level of GAS5 and mitochondrial ROS. The JC- 1 assay findings indicated that corticosterone stimulation suppressed the MtMP, as noted in the JC-1 aggregate-monomer ratio. However, the GAS5 knockdown substantially elevated the MtMP (Fig. 7F). Our results also revealed that the expression of NRF2 and its downstream protein $\mathrm{HO}-1$ were decreased by corticosterone stimulation, which was recovered by GAS5 knockdown (Fig. 7G). Collectively, these results suggested that GAS5 knockdown alleviated microglial activation and neuronal mitochondrial dysfunction in vitro.

\section{GAS5 Suppressed the Expression of NRF2 and SOCS3 Via EZH2-Mediated Epigenetic Repression}

We next investigated the regulation of the expression of SOCS3 and NRF2 by GAS5. GAS5 was silenced by shRNA in HAPI and PC-12 cells. Consistent with our expectations, the expression of SOCS3 in HAPI cells (Fig. 8A and B) and NRF2 in PC-12 cells (Fig. 8C and D) 
was upregulated upon GAS knockdown. GAS5 mediated gene suppression by binding with EZH 2 and facilitating EZH2-mediated epigenetic repression [30]. In the present study, RNA immunoprecipitation (RIP) and RNA pulldown assays revealed GAS5 directly bound with EZH2 in HAPI and PC-12 cells (Fig. 8E to 8H). CHIP-qPCR was performed to validate the enrichment of EZH2 and $\mathrm{H} 3 \mathrm{~K} 27 \mathrm{me} 3$ in the promoter region of SOCS3 or NRF2, which revealed that GAS5 knockdown significantly attenuated the enrichment of EZH2 and H3K27me3 in the promoter region of SOCS3 in HAPI cells (Fig. 8I). Similarly, GAS5 knockdown reduced the enrichment of EZH2 and $\mathrm{H} 3 \mathrm{~K} 27 \mathrm{me} 3$ in the promoter region of NRF2 in PC-12 cells (Fig. 8J). Collectively, these results indicated that GAS5 might suppress the expression of NRF2 and SOCS3 via EZH2-mediated transcriptional repression.

\section{SOCS3 or NRF2 Knockdown Compromised the Protective Effects of Rg1 in HAPI and PC-12 In Vitro Model}

To validate NRF2 or SOCS3 directly mediated the protective effect of $\operatorname{Rg} 1$ on mitochondrial dysfunction and repress microglial activation in vitro model, we applied shRNA to knockdown SOCS3 in HAPI cells and NRF2 in PC-12 cell before stimulation and $\operatorname{Rg} 1$ treatment. As shown in Fig. 9A, Rg1 potentially reduced LPS induced releasing of cytokines while SOCS3 knockdown reversed the effect of $\operatorname{Rg} 1$ on cytokine release. Consistently, we observed that SOCS3 compromised the reduction of COX-2 and iNOS by Rg1 (Fig. 9B). In PC-12 cells, NRF2 knockdown was verified to reverse the protective effect of $\operatorname{Rg} 1$ on intracellular ROS and mitochondrial potential (Fig. 9C and 9D). Western blots in Fig. 9E also indicated that NRF2 knockdown sharply dropped the expression of HO-1 and NRF2, which was restored by $\mathrm{Rg} 1$ in corticosterone stimulated PC-12 cells. Collectively, these results suggested that $\operatorname{Rg} 1$ alleviated microglial activation and neuronal mitochondrial dysfunction at least partly dependent on upregulation of SOCS3 and NRF2.

\section{Discussion}

Depression is a severe mental disorder characterized by depressed mood, retardation of thoughts, and loss of voluntary activity. For a long time, the pathogenesis of depression has remained unclear; various factors, including neuroendocrine, neuroimmunology, genetics, and social influences, may be involved in its pathogenesis [2]. In the present study, our results revealed the anti-depressive effect of $\operatorname{Rg} 1$ in the depression model. $\operatorname{Rg} 1$ treatment attenuated microglial activation and improved mitochondrial dysfunction, thereby alleviating depression-like behaviours by downregulating
lncRNA GAS5. GAS5 inhibition exhibited a similar protective effect of $\operatorname{Rg} 1$ treatment. Mechanically, GAS5 inhibition upregulated the expressions of NRF2 and SOCS3, which EZH2 and GAS5 suppressed. In the present study, the mechanism of the anti-depressive effect of RG1 was deeply explored, providing a theoretical basis for the possible application of RG1 in the treatment of depression.

The anti-inflammatory effects of $\operatorname{Rg} 1$ have been validated in various neurological diseases. Rg1-ameliorated chemotherapy-induced cognitive dysfunction by suppressing microgliamediated neuroinflammation [31]. In Parkinson's disease, $\mathrm{Rg} 1$ reduces LPS-induced neuroinflammation via G-protein coupled oestrogen receptor [32]. Moreover, Zhang et al. demonstrated that $\mathrm{Rg} 1$ abrogated microglial activation in the posttraumatic stress disorder model [33]. In the present study, we verified the anti-inflammatory role of $\operatorname{Rg} 1$ in the depression model. The treatment of $\mathrm{Rg} 1$ reduced IBA-1 positive cell numbers in the hippocampus and abrogated the release of pro-inflammatory cytokines. This finding was consistent with previous studies, which confirmed the anti-inflammatory effect of $\operatorname{Rg} 1$ in the depression model [20, 34]. Additionally, $\mathrm{Rg} 1$ is involved in the regulation of mitochondria function. $\mathrm{Xu}$ et al. suggested that Rg1 improved mitochondrial function by suppressing oxygen-glucose deprivation-induced injury in astrocytes [35]. Another report revealed that $\operatorname{Rg} 1$ repressed isoflurane-induced caspase- 3 activation by preventing mitochondrial dysfunction [36]. As mentioned previously, mitochondrial dysfunction was generally found in the depression model. In the present study, we first validated the beneficial effect of $\operatorname{Rg} 1$ on mitochondria in the depression model.

Some studies have revealed that $\operatorname{Rg} 1$ exerts its function via the regulation of lncRNA. A previous report has suggested that Rg1 inhibits high glucose-induced fibrosis by regulating lncRNA RP11-982M15.8 [37]. To the best of our knowledge, we are the first to validate that Rg1 regulated lncRNA GAS5. A functional study revealed that GAS5 exhibits a protective effect in the depression model by downregulating GAS5 expression. The inhibition of GAS5 revealed an antiinflammatory impact on spinal cord ischemia reperfusion [38] and in oxidative stress induced by oxidized low-density lipoprotein [39]. Moreover, GAS5 is involved in mitochondria-mediated apoptosis, indicating that GAS5 might regulate mitochondrial function [40]. In the present study, we confirmed that the inhibition of GAS5 alleviated microglial activation and mitochondrial dysfunction, thereby ameliorating depression-like behaviours in the depression model. This finding was consistent with the previous study [27].

Moreover, the present study revealed the detailed mechanism of GAS5 in depression. We found that GAS5 was directly bound with EZH2 and mediated the suppression of NRF2 and SOCS3 by EZH2. This finding deepened the understanding of the mechanism by which GAS5 exhibits its detrimental role in depression. However, we failed 

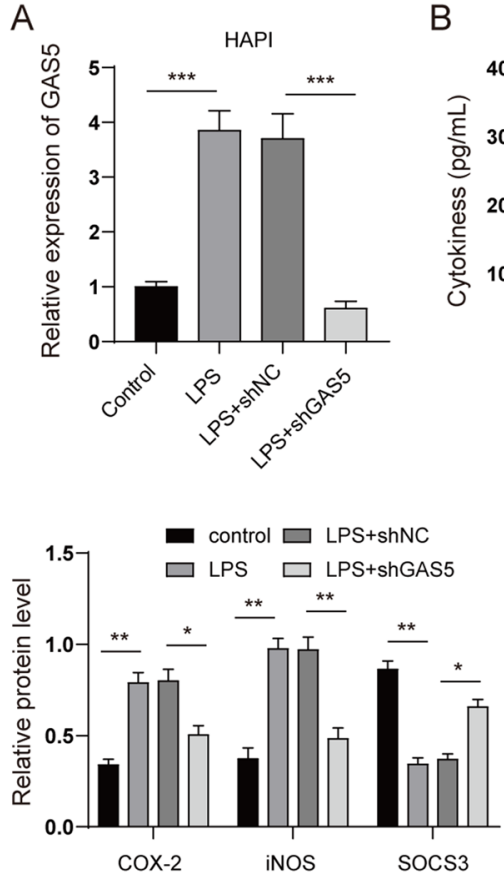

$\mathrm{F}$

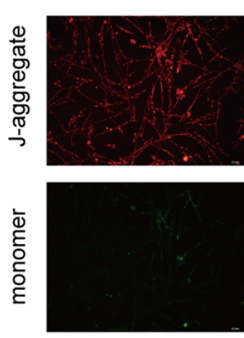

Control
B

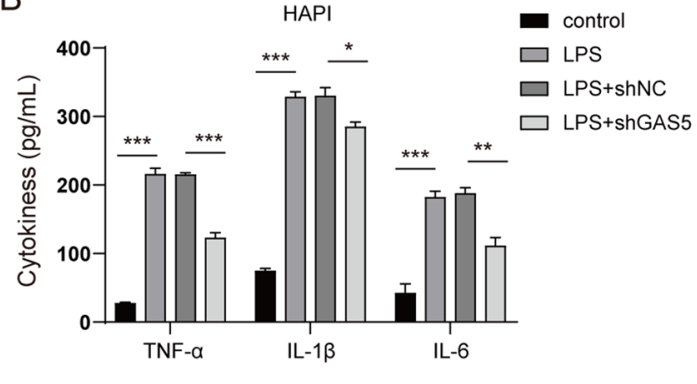

C

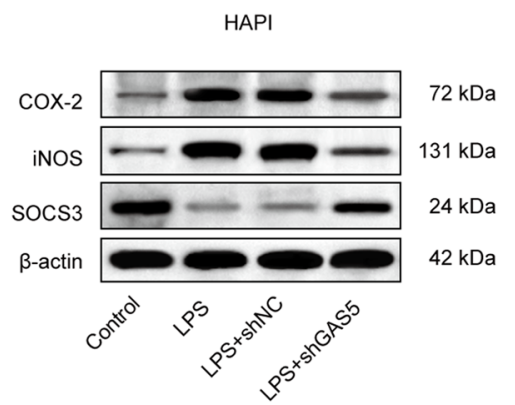

D

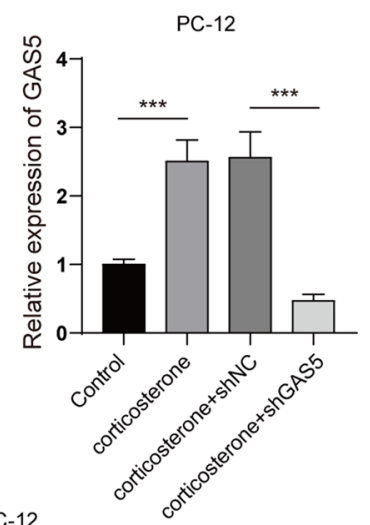

E

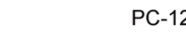

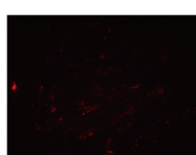

Control

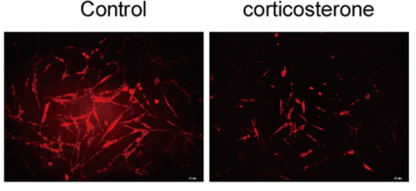

corticosterone+shNC corticosterone+shGAS5
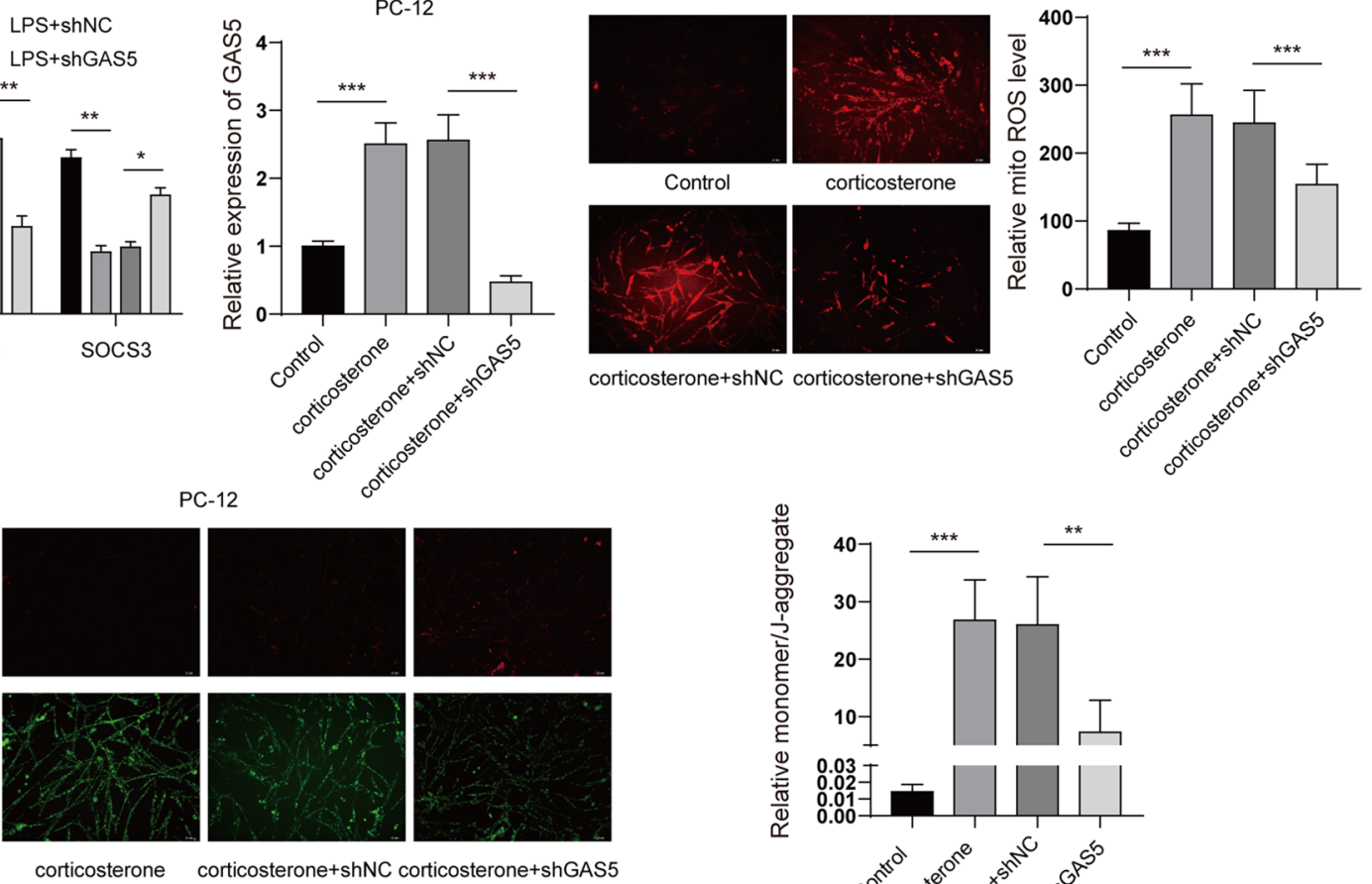

corticosterone corticosterone+shNC corticosterone+shGAS5

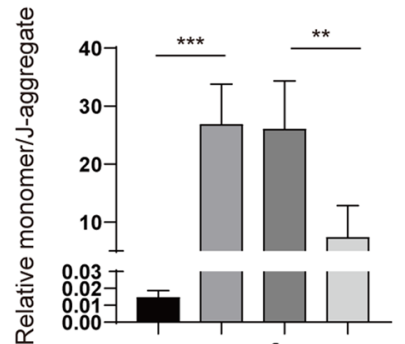

G
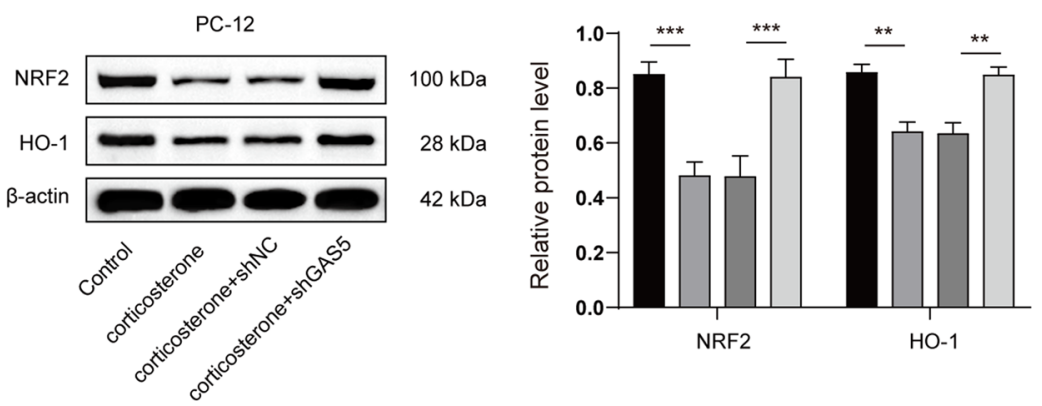

m control

$\square$ corticosterone

$\square$ corticosterone+shNC

$\square$ corticosterone+shGAS5

Fig. 7 GAS5 knockdown alleviated microglial activation and neuronal mitochondria dysfunction in vitro. $A$ and $B$ Rat microglia cell line HAPI was transfected with GAS5 shRNA and $48 \mathrm{~h}$ after transfection, HAPI cells were stimulated with LPS $(1 \mu \mathrm{M})$ for $24 \mathrm{~h}$. A The expression of GAS5 was detected by qRT-PCR. B The release of pro-inflammatory cytokines was detected by ELISA. C The protein levels of COX-2, iNOS, and SOCS3 in HAPI cells were detected by western blots. D to G PC-12 cells were transfected with GAS5
shRNA and $48 \mathrm{~h}$ after transfection, PC-12 cells were subjected to corticosterone $(400 \mu \mathrm{M})$ stimulation for $24 \mathrm{~h}$. D The expression of GAS5 was detected by qRT-PCR. E Mitochondrial ROS in PC-12 cells was detected by the mitoSOX kit. F Mitochondrial membrane potential in PC-12 cells was detected by the JC-1 assay. G The expression of NRF2 and HO-1 in PC-12 cells was detected by western blots. ${ }^{*} P<$ $0.05 ; * * P<0.01 ; * * * P<0.001$ 
to validate how $\operatorname{Rg} 1$ regulated GAS5 and whether $\operatorname{Rg} 1$ could regulate other lncRNA involved in the pathology of depression. We must focus on this obstacle in our further investigation.

The zeste homolog 2 enhancer (EZH2) is the major subunit of polycomb repressive complex 2 (PRC2). EZH2 suppresses the downstream gene expression via the methylation of histone 3 on lysine 27 (H3K27me3) in the promoter of the target gene [41]. The function of EZH2 is generally regulated by lncRNA and circular RNA. GAS5 inhibits the level of ATP-binding cassette transporter A1
(ABCA1) by directly binding with EZH2 and facilitating the methylation of $\mathrm{H} 3 \mathrm{~K} 27 \mathrm{me} 3$ modification in the promoter region of ABCA1 [42]. This finding was supported by other studies, which indicated that GAS5 suppresses the expression of MMP9 by recruiting EZH2 to the promoter region of MMP9 [30]. Consistently, in the present study, we also validated that GAS5 facilitated the function of EZH2 in the suppression of NRF2 and SOCS3. GAS5 knockdown decreased EZH2 and H3K27me3 expressions in the promoter region of NRF2 and SOCS3 and thereby rescued the expression of NRF2 and SOCS3. However,
A

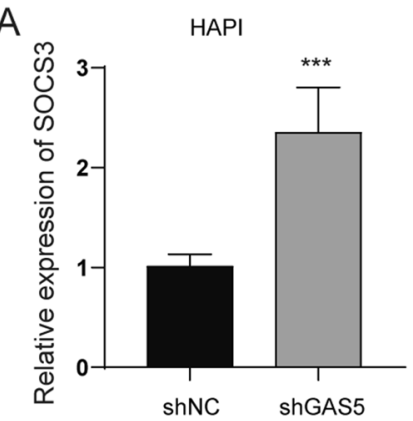

$\mathrm{D}$
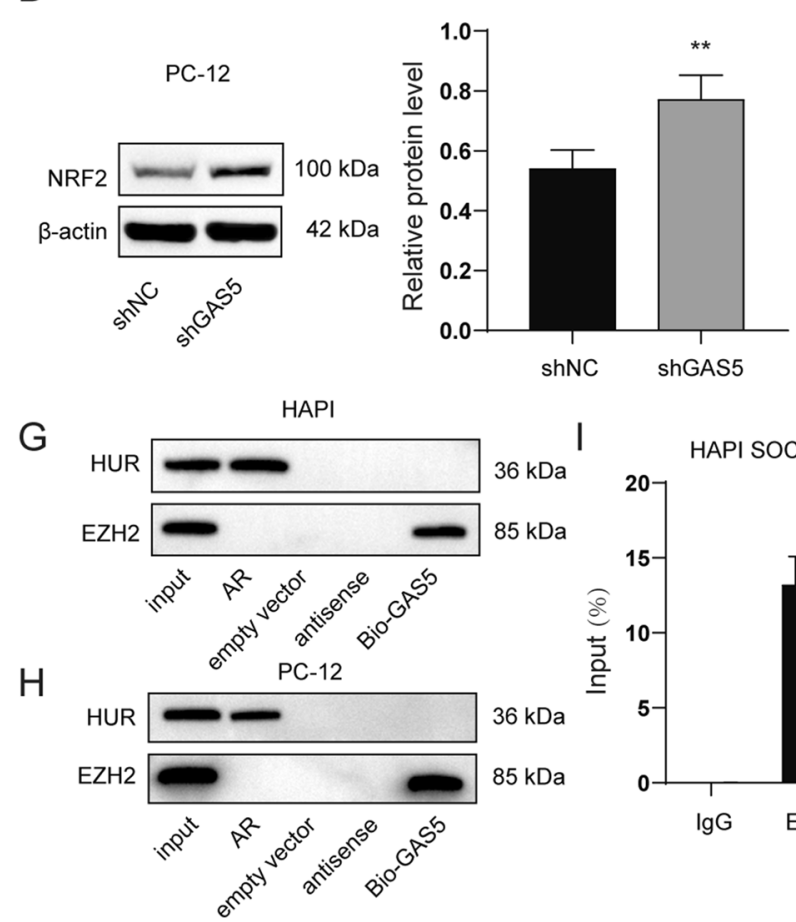

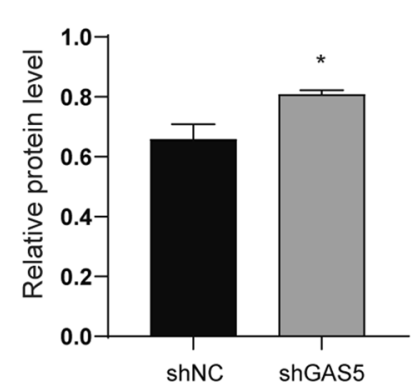

E

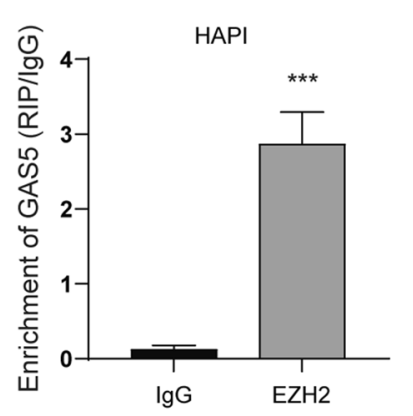

C

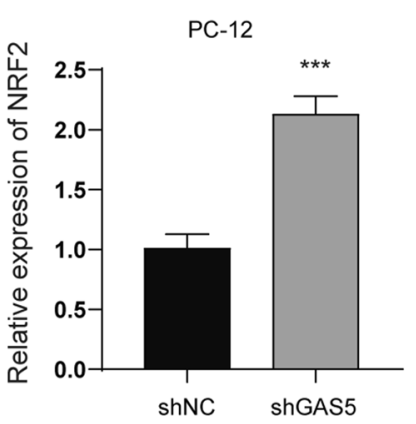

$\mathrm{F}$

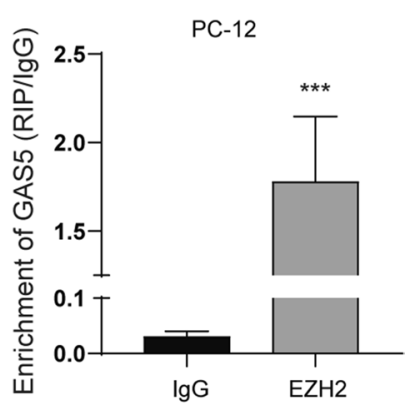

I HAPI SOCS3 promoter J

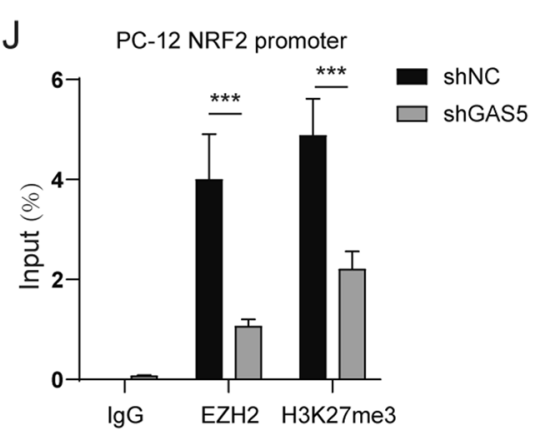

Fig. 8 GAS5 suppressed the expression of NRF2 and SOCS3 via EZH2-mediated epigenetic repression. HAPI cells and PC-12 cells was transfected with GAS5 shRNA. 48 after transfection, followed experiments were performed. A and $\mathbf{B}$ The level of SOCS3 in HAPI cells was detected by qPCR (A) and western blots (B). C and D The level of NRF2 in PC-12 cells was detected by qPCR (C) and western blots (D). E and F RIP was performed to validate binding between
GAS5 and EZH2. G and $\mathbf{H}$ RNA pull-down assay was performed to validate binding between GAS5 and EZH2. I CHIP-qPCR was performed to validate the enrichment of EZH2 and H3K27me3 in the promoter region of SOCS3 in HAPI cells. J CHIP-qPCR was performed to validate the enrichment of EZH2 and $\mathrm{H} 3 \mathrm{~K} 27 \mathrm{me} 3$ in the promoter region of NRF2 in PC-12 cells. ${ }^{*} P<0.05$; $* * P<0.01$; $* * * P<0.001$ 
A
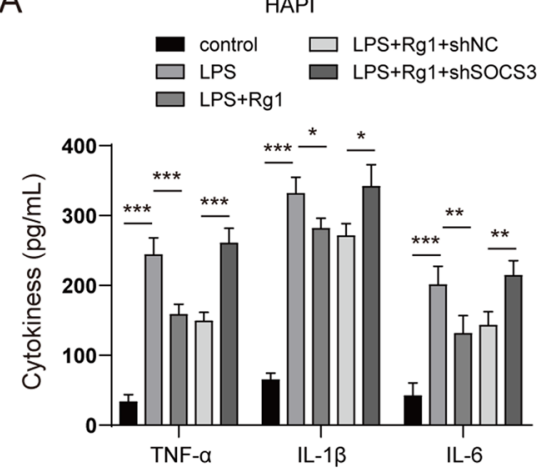

C

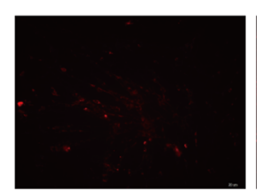

Control

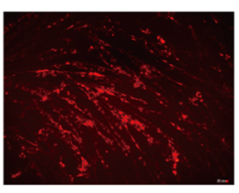

corticosterone

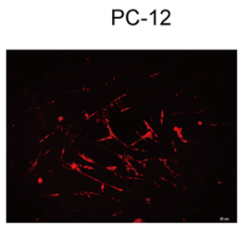

corticosterone+Rg1

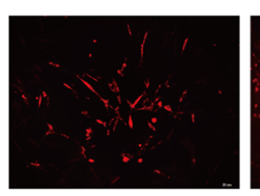

corticosterone+Rg1+shNC corticosterone+Rg1+shNRF2
B
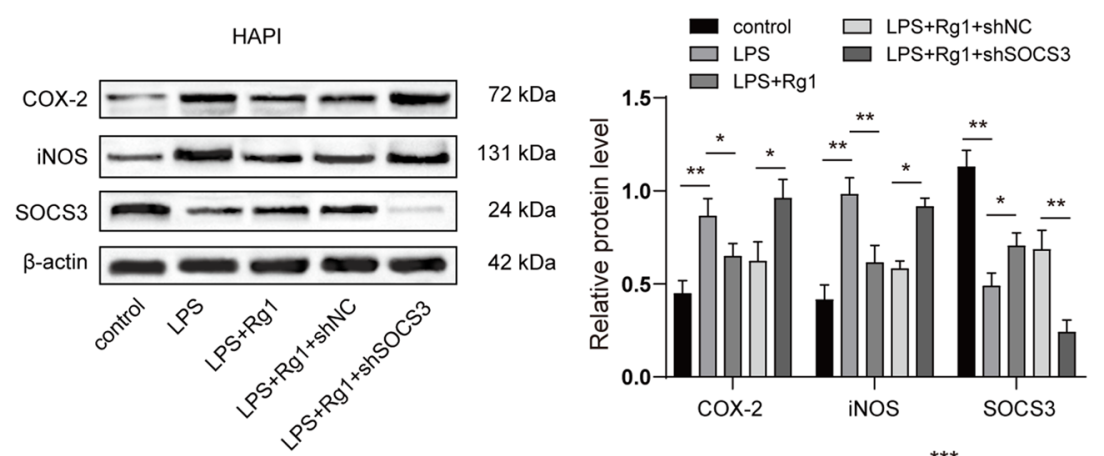

D

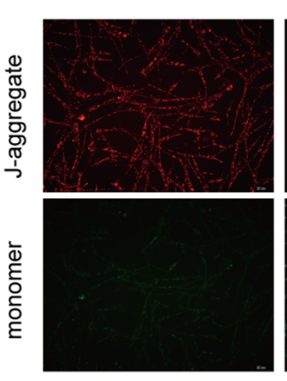

Control

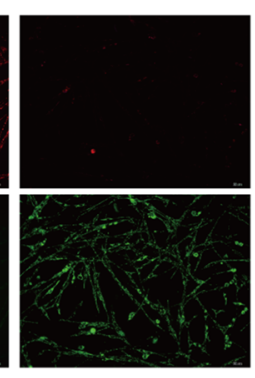

corticosterone

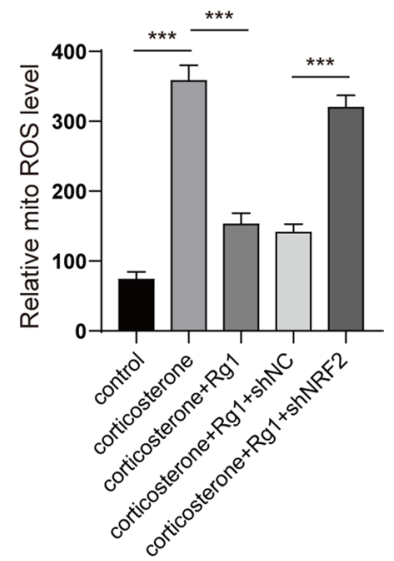

E

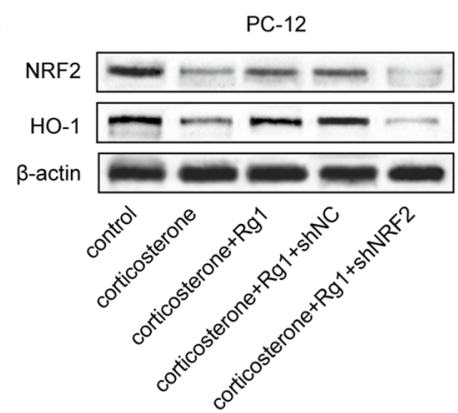

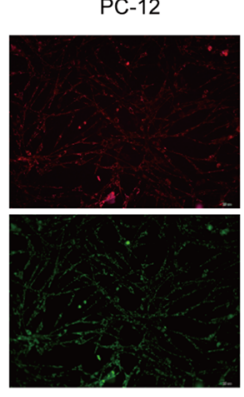

corticosterone+Rg1 corticosterone+Rg1+shNC corticosterone+Rg1+shNRF2
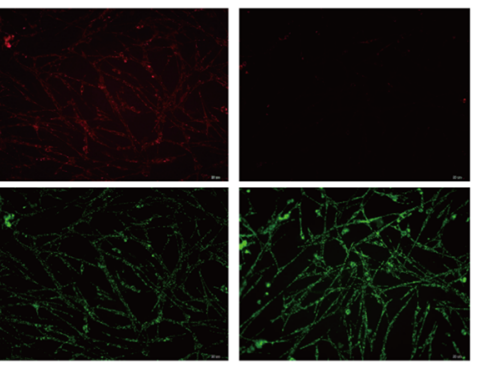

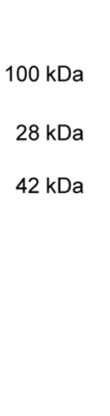

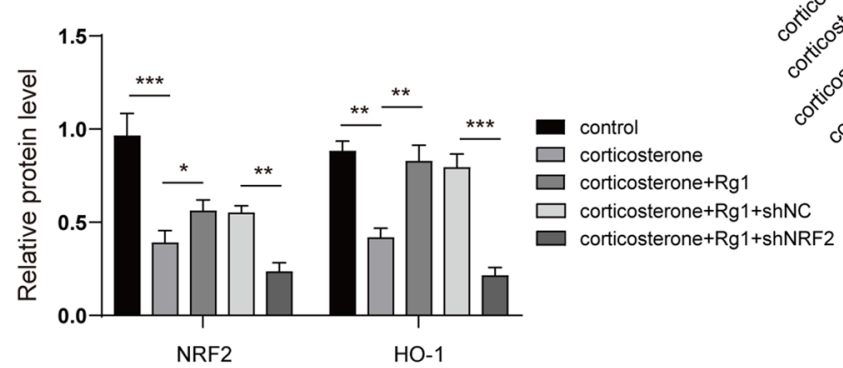

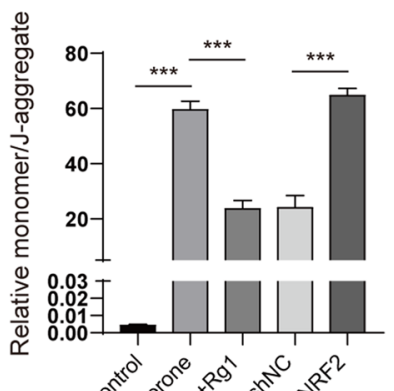

Fig. 9 SOCS3 or NRF2 knockdown compromised the protective effects of Rg1 in HAPI and PC-12 in vitro model. A and B HAPI cells were transfected with SOCS3 shRNA. Forty-eight hours after transfection, the cells were stimulated with LPS $(1 \mu \mathrm{M})$ for $2 \mathrm{~h}$, followed by co-incubation with $\operatorname{Rg} 1(5 \mu \mathrm{M}, 10 \mu \mathrm{M}, 20 \mu \mathrm{M})$ for a total $24 \mathrm{~h}$ LPS and $\operatorname{Rg} 1$ (A). The release of pro-inflammatory cytokines in supernatant of HAPI cells was detected by ELISA. B The protein level of COX-2, iNOS, and SOCS3 in HAPI cells was detected by western blots. $\mathbf{C}$ to $\mathbf{E}$ PC-12 cells were transfected with NRF2
shRNA. Forty-eight hours after transfection, the cells were stimulated with corticosterone $(400 \mu \mathrm{M})$ for $2 \mathrm{~h}$, followed by co-incubation with $\operatorname{Rg} 1(5 \mu \mathrm{M}, 10 \mu \mathrm{M}, 20 \mu \mathrm{M})$ for a total $24 \mathrm{~h}$ subjected to corticosterone stimulation and $\mathrm{Rg} 1$ treatment. C Mitochondrial ROS in PC-12 cells was detected by the mitoSOX kit. D Mitochondrial membrane potential in PC-12 cells was detected by the JC-1 assay. E The expression of NRF2 and HO-1 in PC-12 cells was detected by western blots. $* P<0.05 ; * * P<0.01 ; * * * P<0.001$ 
other studies have revealed a contentious relationship between GAS5 and EZH2. A study by Zhu et al. suggested that GAS5 suppresses Th1 differentiation by inhibiting the expression of EZH2 [43]. Another study further validated that GAS5 decreased the expression of EZH2 via recruiting E2F4 to the promoter region of EZH2 [44]. This contrary conclusion might be due to the different regulations of GAS5 on EZH2 in various diseases. EZH2 mediates neuroinflammation and depression-like behaviours [45], which is in line with the findings of the present study. However, the functional role of EZH2 in depression should be further investigated in future work.

In the present study, we revealed the expression of two downstream genes, NRF2 and SOCS3, was inhibited by the GAS5/ EZH2 axis in the depression model. The epigenetic suppression of SOCS3 by EZH2 was validated in a previous study [28], which was also supported by the findings of our present study. SOCS3 negatively regulates microglial activation in neuropathic pain [46]. Interestingly, the inhibition of EZH2 in a depression model elevated the expression of SOCS3 [45], which suggested that SOCS3 might regulate microglial activation in depression. However, more studies are warranted to confirm this hypothesis. NRF2 is a major transcription factor responsible for the antioxidant effect in cells [47]. NRF2 mediated the protective effect of adipose-derived mesenchymal stem cell (ADSC) [48] and melatonin [12] on depression-like behaviours. Previous work has also indicated that NRF2 has a protective effect on mitochondrial dysfunction [49]. Consistent with our findings, another research also validated that NRF2 was downregulated in a depression model and restored by Rg1 treatment [34]. Therefore, we speculated that $\operatorname{Rg} 1 /$ GAS5 regulates mitochondrial dysfunction in depression by regulating the expression of NRF2. More studies are warranted to validate this hypothesis.

In conclusion, we verified the protective role of $\operatorname{Rg} 1$ in a depression model. $\operatorname{Rg} 1$ attenuated microglial activation and improved mitochondrial dysfunction by downregulating the expression of GAS5. We also validated the antidepressive function of GAS5 knockdown in our present study. Mechanically, GAS5 regulated microglial activation and mitochondrial dysfunction via the EZH2-mediated epigenetic suppression of NRF2 and SOCS3. Our result might provide novel insights into understanding the role of $\operatorname{Rg} 1$ in the treatment of depression.

Author Contribution Junnan Li: concepts, design, funding acquisition, writing-original draft preparation; Wei Gao: experimental studies; Zhonghui Zhao: experimental studies; YannanLi: data acquisition, data analysis; Lixuan Yang: experimental studies; Wei Wei: data acquisition, data analysis; Feifei Ren: data acquisition, data analysis; Yang Li: data acquisition, data analysis; Yao Yu: design, funding acquisition; Wenzhe Duan: data acquisition, data analysis; Jingchun Li: experimental studies; Baoan Dai: experimental studies; Rongjuan Guo: supervision, writing —reviewing and editing; all the authors approved for the final version.
Funding This work was supported by the National Natural Science Foundation of China "Study on the Effector Mechanism of "Xingpi Jieyu method" on Depression Based on the Variation in Intestinal Microflora" (NO.81874422).

Data Availability The datasets used or analyzed during the current study are available from the corresponding author on reasonable request.

\section{Declarations}

Ethics Approval and Consent to Participate All animal experiments were strictly followed the GUIDELINES of the China-Japan Friendship Hospital on the care and use of laboratory animals, and the study was approved by the Ethics Committee of China-Japan Friendship Hospital.

Consent for Publication Not applicable.

Conflict of Interest The authors declare no competing interests.

Open Access This article is licensed under a Creative Commons Attribution 4.0 International License, which permits use, sharing, adaptation, distribution and reproduction in any medium or format, as long as you give appropriate credit to the original author(s) and the source, provide a link to the Creative Commons licence, and indicate if changes were made. The images or other third party material in this article are included in the article's Creative Commons licence, unless indicated otherwise in a credit line to the material. If material is not included in the article's Creative Commons licence and your intended use is not permitted by statutory regulation or exceeds the permitted use, you will need to obtain permission directly from the copyright holder. To view a copy of this licence, visit http://creativecommons.org/licenses/by/4.0/.

\section{References}

1. Walker AK et al (2019) Leucine competes with kynurenine for blood-to-brain transport and prevents lipopolysaccharideinduced depression-like behavior in mice. Mol Psychiatry 24(10):1523-1532

2. Menard C, Hodes GE, Russo SJ (2016) Pathogenesis of depression: Insights from human and rodent studies. Neuroscience 321:138-162

3. Seo JS et al (2017) Cellular and molecular basis for stress-induced depression. Mol Psychiatry 22(10):1440-1447

4. Hofmann SG et al (2017) Effect of treatments for depression on quality of life: a meta-analysis. Cogn Behav Ther 46(4):265-286

5. Wolf SA, Boddeke HW, Kettenmann H (2017) Microglia in Physiology and Disease. Annu Rev Physiol 79:619-643

6. Block ML, Zecca L, Hong JS (2007) Microglia-mediated neurotoxicity: uncovering the molecular mechanisms. Nat Rev Neurosci 8(1):57-69

7. Hansen DV, Hanson JE, Sheng M (2018) Microglia in Alzheimer's disease. J Cell Biol 217(2):459-472

8. Ho MS (2019) Microglia in Parkinson's disease. Adv Exp Med Biol 1175:335-353

9. Deng SL, Chen JG, Wang F (2020) Microglia: a central player in depression. Curr Med Sci 40(3):391-400

10. Liu LL et al (2019) Sex differences in depressive-like behaviour may relate to imbalance of microglia activation in the hippocampus. Brain Behav Immun 81:188-197

11. Bassett B et al (2021) Minocycline alleviates depressionlike symptoms by rescuing decrease in neurogenesis in dorsal 
hippocampus via blocking microglia activation/phagocytosis. Brain Behav Immun 91:519-530

12. Arioz BI et al (2019) Melatonin attenuates LPS-induced acute depressive-like behaviors and microglial NLRP3 inflammasome activation through the SIRT1/Nrf2 pathway. Front Immunol 10:1511

13. Nunnari J, Suomalainen A (2012) Mitochondria: in sickness and in health. Cell 148(6):1145-1159

14. Bansal Y, Kuhad A (2016) Mitochondrial dysfunction in depression. Curr Neuropharmacol 14(6):610-618

15. Tanaka M et al (1996) Accumulation of deletions and point mutations in mitochondrial genome in degenerative diseases. Ann N Y Acad Sci 786:102-111

16. Wang Y et al (2019) Mitochondrial transplantation attenuates lipopolysaccharide- induced depression-like behaviors. Prog Neuropsychopharmacol Biol Psychiatry 93:240-249

17. Chu SF et al (2019) Ginsenoside Rg1 protects against ischemic/ reperfusion-induced neuronal injury through miR-144/Nrf2/ARE pathway. Acta Pharmacol Sin 40(1):13-25

18. Jiang W et al (2015) Ginsenoside Rg1 ameliorates motor function in an animal model of Parkinson's disease. Pharmacology 96(1-2):25-31

19. Quan Q et al (2020) Ginsenoside Rg1 reduces \&beta;amyloid levels by inhibiting CD\&Kappa;5induced PPAR\&gamma; phosphorylation in a neuron model of Alzheimer's disease. Mol Med Rep 22(4):3277-3288

20. Li Y et al (2020) Ginsenoside-Rg1 rescues stress-induced depression-like behaviors via suppression of oxidative stress and neural inflammation in rats. Oxid Med Cell Longev 2020:2325391

21. Jiang B et al (2012) Antidepressant-like effects of ginsenoside Rg1 are due to activation of the BDNF signalling pathway and neurogenesis in the hippocampus. Br J Pharmacol 166(6):1872-1887

22. Yu H et al (2018) Ginsenoside Rg1 prevents chronic stressinduced depression-like behaviors and neuronal structural plasticity in rats. Cell Physiol Biochem 48(6):2470-2482

23. Issler $\mathrm{O}$ et al (2020) Sex-specific role for the long non-coding RNA LINC00473 in depression. Neuron 106(6):912-9265 e5

24. Seki T et al (2019) Altered expression of long non-coding RNAs in patients with major depressive disorder. J Psychiatr Res 117:92-99

25. Gu XH et al (2020) Long non-coding RNA uc.80- overexpression promotes M2 polarization of microglias to ameliorate depression in rats. IUBMB Life 72(10):2194-2203

26. Huan $\mathrm{Z}$ et al (2021) lncRNA MIR155HG alleviates depressionlike behaviors in mice by regulating the miR-155/BDNF axis. Neurochem Res 46(4):935-944

27. Wu Y et al (2021) Downregulation of 1ncRNA GAS5 alleviates hippocampal neuronal damage in mice with depression-like behaviors via modulation of MicroRNA-26a/EGR1 axis. J Stroke Cerebrovasc Dis 30(3): 105550

28. Zhang X et al (2018) Macrophage/microglial Ezh2 facilitates autoimmune inflammation through inhibition of Socs3. J Exp Med 215(5):1365-1382

29. Li Z et al (2014) The polycomb group protein EZH2 inhibits lung cancer cell growth by repressing the transcription factor Nrf2. FEBS Lett 588(17):3000-3007

30. Zhang L, Zhao S, Zhu Y (2020) Long non-coding RNA growth arrest-specific transcript 5 alleviates renal fibrosis in diabetic nephropathy by downregulating matrix metalloproteinase 9 through recruitment of enhancer of zeste homolog 2. FASEB J 34(2):2703-2714

31. Shi DD et al (2019) Ginsenoside Rg1 prevents chemotherapyinduced cognitive impairment: associations with microgliamediated cytokines, neuroinflammation, and neuroplasticity. Mol Neurobiol 56(8):5626-5642
32. Gao XQ et al (2019) Ginsenoside Rg1 exerts anti-inflammatory effects via $G$ protein-coupled estrogen receptor in lipopolysaccharide-induced microglia activation. Front Neurosci 13:1168

33. Zhang Z et al (2021) Ginsenoside Rg1 prevents PTSD-like behaviors in mice through promoting synaptic proteins, reducing Kir4.1 and TNF-alpha in the hippocampus. Mol Neurobiol 58(4):1550-1563

34. Fan C et al (2018) Neuroprotective effects of ginsenoside-Rg1 against depression-like behaviors via suppressing glial activation, synaptic deficits, and neuronal apoptosis in rats. Front Immunol 9:2889

35. Xu M et al (2019) Ginsenosides Rb1 and Rg1 protect primary cultured astrocytes against oxygen-glucose deprivation/reoxygenation-induced injury via improving mitochondrial function. Int $\mathrm{J}$ Mol Sci 20(23):6086

36. Miao HH et al (2015) Ginsenoside $\mathrm{Rg} 1$ attenuates isofluraneinduced caspase- 3 activation via inhibiting mitochondrial dysfunction. Biomed Environ Sci 28(2):116-126

37. Xue LP et al (2018) Rg1 inhibits high glucose-induced mesenchymal activation and fibrosis via regulating miR-2113/RP11982M15.8/Zeb1 pathway. Biochem Biophys Res Commun 501(4):827-832

38. Zhang Z et al (2021) Downregulation of LncRNA Gas5 inhibits apoptosis and inflammation after spinal cord ischemia-reperfusion in rats. Brain Res Bull 168:110-119

39. Zhang Y et al (2021) GAS5 knockdown suppresses inflammation and oxidative stress induced by oxidized low-density lipoprotein in macrophages by sponging miR-135a. Mol Cell Biochem 476(2):949-957

40. Gao J et al (2015) Long non-coding RNA growth arrest-specific transcript 5 is involved in ovarian cancer cell apoptosis through the mitochondria-mediated apoptosis pathway. Oncol Rep 34(6):3212-3221

41. Duan R, Du W, Guo W (2020) EZH2: a novel target for cancer treatment. J Hematol Oncol 13(1):104

42. Meng XD et al (2020) Knockdown of GAS5 inhibits atherosclerosis progression via reducing EZH2-mediated ABCA1 transcription in ApoE(-/-) mice. Mol Ther Nucleic Acids 19:84-96

43. Zhu X et al (2020) Exosomal long non-coding RNA GAS5 suppresses Th1 differentiation and promotes Th2 differentiation via downregulating EZH2 and T-bet in allergic rhinitis. Mol Immunol 118:30-39

44. Xu W et al (2020) Long non-coding RNA GAS5 accelerates oxidative stress in melanoma cells by rescuing EZH2-mediated CDKN1C downregulation. Cancer Cell Int 20:116

45. Wang W et al (2020) EZH2 is involved in vulnerability to neuroinflammation and depression-like behaviors induced by chronic stress in different aged mice. J Affect Disord 272:452-464

46. Zheng Y, Hou X, Yang S (2019) Lidocaine potentiates SOCS3 to attenuate inflammation in microglia and suppress neuropathic pain. Cell Mol Neurobiol 39(8):1081-1092

47. Bellezza I et al (2018) Nrf2-Keap1 signaling in oxidative and reductive stress. Biochim Biophys Acta Mol Cell Res 1865(5):721-733

48. Huang X et al (2020) Adipose-derived mesenchymal stem cells protect against CMS-induced depression-like behaviors in mice via regulating the Nrf2/HO-1 and TLR4/NF-kappaB signaling pathways. Acta Pharmacol Sin 41(5):612-619

49. Abdalkader M et al (2018) Targeting Nrf2 to suppress ferroptosis and mitochondrial dysfunction in neurodegeneration. Front Neurosci 12:466

Publisher's Note Springer Nature remains neutral with regard to jurisdictional claims in published maps and institutional affiliations. 